\title{
MATCHING OF ASYMPTOTIC EXPANSIONS FOR WAVES PROPAGATION IN MEDIA WITH THIN SLOTS II: THE ERROR ESTIMATES
}

\author{
Patrick Joly ${ }^{1}$ and SÉBAstien Tordeux ${ }^{2}$
}

\begin{abstract}
We are concerned with a $2 \mathrm{D}$ time harmonic wave propagation problem in a medium including a thin slot whose thickness $\varepsilon$ is small with respect to the wavelength. In a previous article, we derived formally an asymptotic expansion of the solution with respect to $\varepsilon$ using the method of matched asymptotic expansions. We also proved the existence and uniqueness of the terms of the asymptotics. In this paper, we complete the mathematical justification of our work by deriving optimal error estimates between the exact solutions and truncated expansions at any order.
\end{abstract}

Mathematics Subject Classification. 35J05, 34E05, 78A45, 78A50.

Received February 7, 2007. Revised September 20, 2007.

\section{INTRODUCTION}

Many practical applications concerning time harmonic electromagnetic or acoustic wave propagation involve structures with at least one space dimension of characteristic length $\varepsilon$ which is very small with respect to the wave length $\lambda$. In this paper, we consider 2D thin slots which typically correspond to the geometry of Figure 1.

One interesting situation in applications corresponds to:

$$
\lambda / 1000<\varepsilon<\lambda / 10, \quad \varepsilon<L / 10, \quad \text { and } \quad \lambda / 10<L<10 \lambda,
$$

where $\lambda$ is the wave length, $\varepsilon$ is the width of the slot, and $L$ the length of the slot. This is typical for microwave shielding of thin slots [13] or flanged waveguide antennas [11] (see [20] for more examples).

For numerical simulations of wave propagation in media with thin slots, a natural idea is to derive an approximate "1D-2D" model: a 1D model for the propagation inside the slot and a 2D model for the rest of the computational domain. The 1D model is posed on the curve that materializes the limit of the slot when $\varepsilon$ goes to 0 . The main difficulty consists in finding a good method for coupling the two models. Such models have been designed in the engineering literature (see $[2,6,7,19,20]$ for a review) and are commonly used in various computational codes. However, the complete understanding and evaluation of such models suffer, to our opinion, from a lack of mathematical analysis. In [9] we considered the case of the scalar Helmholtz

\footnotetext{
Keywords and phrases. Slit, slot, wave equation, Helmholtz equation, approximate model, matching of asymptotic expansions.

1 Projet POEMS, Bâtiment 13, INRIA, Domaine de Voluceau - Rocquencourt - B.P. 105, 78153 Le Chesnay Cedex, France. patrick.joly@inria.fr

${ }^{2}$ Institut de Mathématiques de Toulouse, Université de Toulouse, 135 avenue de Rangueil, 31077 Toulouse Cedex 4, France. sebastien.tordeux@insa-toulouse.fr
} 


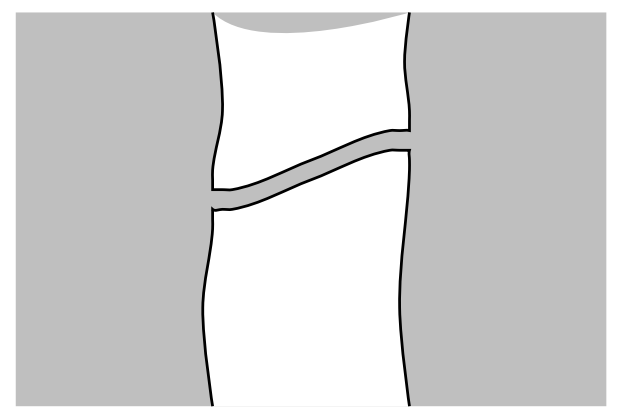

Figure 1. Geometry of the domain of propagation.

equations with homogeneous Neumann boundary conditions (the interesting case from the physical point of view). We proposed a particular $2 \mathrm{D}-1 \mathrm{D}$ technique that we were able to analyze: the obtained accuracy is $O\left(\varepsilon^{2}\right)$.

To improve this accuracy, we need a more complete description of the asymptotic behaviour of the solution. In [10], we derived formal asymptotic expansions of the exact solution using the technique of matched asymptotic expansions (see, for instance, $[5,8,16,24]$ ).

Remark 1.1. An alternative approach is the multiscale technique $[3,14,15,17,25]$. We refer the reader to [23] for the connection between the two approaches.

The objective of the present article is to justify theoretically these formal asymptotic expansions.

The propagation domain is defined by (see Fig. 2)

$$
\begin{gathered}
\bar{\Omega}_{\varepsilon}=\bar{\Omega}_{H} \cup \overline{\Omega_{S}^{\varepsilon}}, \\
\Omega_{S}^{\varepsilon}=\left\{(x, y) \in \mathbb{R}^{2} \mid 0<x \text { and }-\varepsilon / 2<y<\varepsilon / 2\right\}, \\
\Omega_{H}=\left\{\mathbf{x}=(x, y) \in \mathbb{R}^{2} \mid x<0 \text { and } \mathbf{x} \notin \mathcal{B}\right\},
\end{gathered}
$$

where $\mathcal{B}$ is a regular obstacle included in the left half-space $x<0$. We consider the problem ${ }^{1}$

$$
\left\{\begin{array}{l}
\text { Find } u^{\varepsilon} \in H_{l o c}^{1}\left(\Omega_{\varepsilon}\right) \text { outgoing solution of } \\
\Delta u^{\varepsilon}+\omega^{2} u^{\varepsilon}=-f \quad \text { in } \Omega_{\varepsilon} \\
\frac{\partial u^{\varepsilon}}{\partial n}=0 \quad \text { on } \partial \Omega_{\varepsilon}
\end{array}\right.
$$

where the source term $f \in L^{2}\left(\Omega_{H}\right)$ is compactly supported in the half-space $\Omega_{H}$ and $\omega=2 \pi / \lambda$ is the pulsation. The term outgoing solution refers to a prescribed behavior at infinity, needed for ensuring uniqueness result, namely

- in $\Omega_{H}, u^{\varepsilon}$ satisfies the Sommerfeld radiation condition (see [18] for a review);

- inside the slot, the solution is the superposition of modes which are either evanescent or propagating in the direction $x>0$ (see, for example, [9]).

The outline of the paper is as follows. In Section 2.1, we first recall the results obtained in [10]. Namely, we give three different asymptotic expansions in three regions: the half-space (also called the far-field zone), the slot, and the near-field zone which is a transition zone between the slot and the half-space. The terms of the asymptotic expansions are defined as the solutions of coupled problems that have been proved to be uniquely solvable. In Section 2.2, we state our main results (Thms. 2.2, 2.3, and 2.4) which explain in which sense our

\footnotetext{
${ }^{1}$ We have chosen to use the definition $u \in H_{l o c}^{1}(D) \Longleftrightarrow \varphi u \in H^{1}(D), \forall \varphi \in \mathcal{D}\left(\mathbb{R}^{2}\right)$.
} 

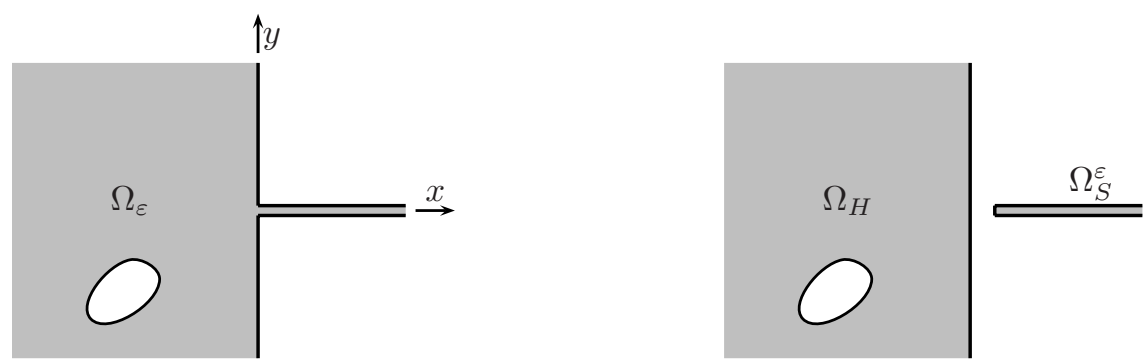

Figure 2. Domain for the model problem.

asymptotic expansions are generalized Taylor expansions of the exact solution. Section 3 is devoted to the proof of the local approximation Theorems 2.2, 2.3, and 2.4, which will appear (Sect. 3.2) as corollaries of a global approximation result (Thm. 3.2, Sect. 3.1). The proof of Theorem 3.2, Section 3.3, relies on a stability property (Lem. 3.4 and Sect. 3.4 for the proof) and on a consistency result (Lem. 3.5 and Sect. 3.5 for the proof). In Section 4 we conclude with some forthcoming and interesting issues.

\section{The Asymptotic EXPANsions And Associated ESTimates}

\subsection{The formal expansions}

\subsubsection{Preliminaries}

Bessel functions and related results. In what follows, we shall make an extensive use of the Bessel functions $J_{p}(z)$ and $Y_{p}(z)$ for $p \in \mathbb{N}$ (see, for example, [12]). We shall use more particularly the generalized Taylor series expansion of these functions (one of their possible definitions)

$$
\left\{\begin{aligned}
J_{p}(z) & =\sum_{l=-\infty}^{+\infty} J_{p, l}\left(\frac{z}{2}\right)^{l} \\
Y_{p}(z) & =\sum_{l=-\infty}^{+\infty} Y_{p, l}\left(\frac{z}{2}\right)^{l}+\sum_{l=-\infty}^{+\infty} \frac{2}{\pi} J_{p, l}\left(\frac{z}{2}\right)^{l} \log \frac{z}{2}
\end{aligned}\right.
$$

where the numbers $J_{p, l}$ are given by

$$
\begin{cases}J_{p, p+l}=0, & \text { if } l<0 \text { or } l \text { odd } \\ J_{p, p+2 l}=\frac{(-1)^{l}}{l !(l+p) !}, & \text { if } l \geq 0\end{cases}
$$

and the numbers $Y_{p, l}$ are given by

$$
\begin{cases}Y_{p,-p+l}=0, & \text { if } l<0 \text { or } l \text { odd, } \\ Y_{p,-p+2 l}=-\frac{1}{\pi} \frac{(p-l-1) !}{l !}, & \text { if } 0 \leqslant l<p, \\ Y_{p, p+2 l}=-\frac{1}{\pi} \frac{(-1)^{l}}{l !(l+p) !}(\psi(l+1)+\psi(l+p+1)), & \text { if } p \leqslant l,\end{cases}
$$


with ( $\gamma$ is the Euler number)

$$
\psi(1)=-\gamma, \quad \psi(k+1)=-\gamma+\sum_{m=1}^{k} \frac{1}{m}, \quad \forall k \in \mathbb{N}^{*}
$$

Next, we introduce two particular sequences of functions $\left(\jmath_{p, l}, y_{p, l}, p \in \mathbb{N}, l \in \mathbb{Z}\right)$, playing a central role in the forthcoming modal expansions, see (2.9) and (2.26). They are identically zero for odd $l$ or $l<0$, and are constructed from the numbers $\left(J_{p, l}, Y_{p, l}\right)$

$$
\left\{\begin{array}{l}
J_{p, l}(\rho, \theta)=J_{p, p+l} \rho^{p+l} \cos p \theta \\
y_{p, l}(\rho, \theta)=\left(Y_{p,-p+l}+\frac{2}{\pi} J_{p,-p+l} \log \rho\right) \rho^{-p+l} \cos p \theta
\end{array}\right.
$$

Local modal expansions of solutions of the Helmholtz equation in the half-space. In this paragraph, we consider particular solutions of Helmholtz equation in the half-space in which the origin $\mathbf{0}$ plays a particular role.

To this end, we introduce $H_{0, l o c}^{1}\left(\Omega_{H}\right)$ the space of " $H_{l o c}^{1}\left(\Omega_{H}\right)$ functions except at the origin"

$$
H_{0, l o c}^{1}\left(\Omega_{H}\right)=\left\{u \in \mathcal{D}^{\prime}\left(\Omega_{H}\right) / \forall \varphi \in C_{00}^{\infty}\left(\Omega_{H}\right), \quad \varphi u \in H^{1}\left(\Omega_{H}\right)\right\},
$$

with $C_{00}^{\infty}\left(\Omega_{H}\right)=\left\{\varphi \in C^{\infty}\left(\overline{\Omega_{H}}\right) / \exists r_{1}>r_{0}>0\right.$ such that $r_{0} \geqslant|\mathbf{x}|$ or $\left.|\mathbf{x}| \geqslant r_{1} \Rightarrow \varphi(\mathbf{x})=0\right\}$.

We are more specifically concerned in a space of $H_{0, l o c}^{1}$ functions which satisfy the homogeneous Helmholtz equation and the Neumann condition only in a neighborhood of the origin. More precisely, we introduce

$$
\left\{\begin{array}{l}
\Omega_{H}^{R}=\left\{\mathbf{x} \in \Omega_{H} \text { and } 0<|\mathbf{x}|<R\right\}, \quad(\Longleftrightarrow 0<r<R, 0<\theta<\pi) \\
\mathcal{V}\left(\Omega_{H}^{R}\right)=\left\{u \in H_{0, l o c}^{1}\left(\Omega_{H}\right) / \Delta u+\omega^{2} u=0 \text { in } \Omega_{H}^{R} \text { and } \frac{\partial u}{\partial \theta}(r, \theta=0, \pi)=0,0<r<R\right\}
\end{array}\right.
$$

with $(r, \theta)$ the polar coordinates so that $x=-r \sin \theta, y=r \cos \theta$. In what follows, $R$ will be chosen small enough in such a way that

$$
(\operatorname{supp} f \cup \overline{\mathcal{B}}) \cap \Omega_{H}^{R}=\emptyset \text {. }
$$

The method of separation of variables in $(r, \theta)$ naturally introduces the functions $(\cos p \theta, p \in \mathbb{N})$, which are the eigenfunctions of the operator $-\mathrm{d}^{2} / \mathrm{d} \theta^{2}$ in the interval $] 0, \pi[$ with Neumann conditions at $\theta=0$ or $\pi$. Any $u \in \mathcal{V}\left(\Omega_{H}^{R}\right)$ admits the following expansion (the convergence holds uniformly in any domain $r_{0}<r<R$ )

$$
\left\{\begin{aligned}
u(r, \theta) & =\sum_{p=0}^{+\infty}\left(\mathcal{L}_{p}^{0}(u) Y_{p}(\omega r) \cos p \theta+\mathcal{L}_{p}^{1}(u) J_{p}(\omega r) \cos p \theta\right), \\
& =\sum_{p=0}^{+\infty} \sum_{l=0}^{+\infty}\left(\mathcal{L}_{p}^{0}(u) y_{p, l}\left(\frac{\omega r}{2}, \theta\right)+\mathcal{L}_{p}^{1}(u) j_{p, l}\left(\frac{\omega r}{2}, \theta\right)\right),
\end{aligned}\right.
$$

where the complex coefficients $\mathcal{L}_{p}^{0}(u)$ and $\mathcal{L}_{p}^{1}(u)$ define linear forms in $\mathcal{V}\left(\Omega_{H}^{R}\right)$ that can be also defined as (setting $\delta_{0}=1$ and $\delta_{p}=2$ for $p>0$ )

$$
\left\{\begin{array}{l}
\mathcal{L}_{p}^{0}(u)=\lim _{r \rightarrow 0} \frac{\delta_{p}}{\pi Y_{p}(\omega r)} \int_{0}^{\pi}[u(r, \theta) \cos p \theta] \mathrm{d} \theta \\
\mathcal{L}_{p}^{1}(u)=\lim _{r \rightarrow 0} \frac{\delta_{p}}{\pi J_{p}(\omega r)} \int_{0}^{\pi}\left[u(r, \theta)-\mathcal{L}_{p}^{0}(u) Y_{p}(\omega r) \cos p \theta\right] \cos p \theta \mathrm{d} \theta .
\end{array}\right.
$$




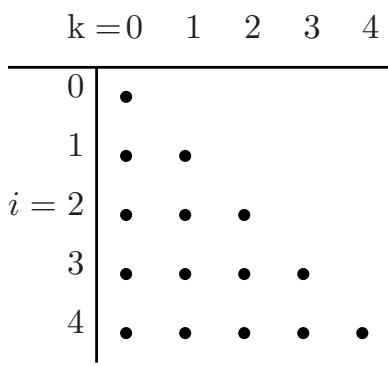

Figure 3. The index set $\mathbf{J}$.

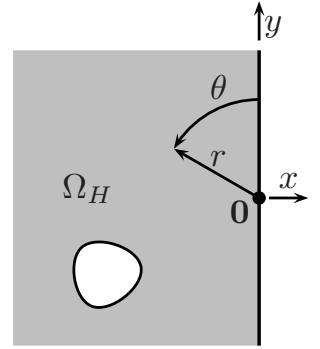

FiguRE 4. Far-field domain.

Remark 2.1. All functions of $\mathcal{V}\left(\Omega_{H}^{R}\right)$ are regular $\left(C^{\infty}\right)$ except in a neighborhood of the origin. These singularities are due to the presence of $Y_{p}(\omega r) \cos p \theta$

$$
Y_{0}(z)=O(\log (z)) \quad \text { and } \quad Y_{p}(z)=O\left(z^{-p}\right), \quad \text { for } z \rightarrow 0
$$

\subsubsection{Definition of the terms of the asymptotic expansions}

Asymptotic expansion of the far-field. By far-field expansion we mean the expansion with respect to $\varepsilon$ of the exact solution $u^{\varepsilon}$ in the far-field domain $\Omega_{H}$ (the half-space without the obstacle $\mathcal{B}$, see Fig. 4) sufficiently far away from the origin. This expansion uses two indices lying in the set (see Fig. 3)

$$
\mathbf{J}=\left\{(i, k) \in \mathbb{N}^{2} / k \leqslant i\right\},
$$

and is of the form

$$
u^{\varepsilon}=\sum_{i=0}^{+\infty} \sum_{k=0}^{i}\left(\frac{\omega \varepsilon}{2}\right)^{i} \log ^{k}\left(\frac{\omega \varepsilon}{2}\right) u_{i}^{k}+o\left(\varepsilon^{\infty}\right), \quad \text { in } \Omega_{H} .
$$

The function $u_{0}^{0} \in H_{l o c}^{1}(\Omega)$ is the limit solution in the half-space

$$
\Delta u_{0}^{0}+\omega^{2} u_{0}^{0}=-f \text { in } \Omega_{H}, \quad \frac{\partial u_{0}^{0}}{\partial n}=0 \text { on } \partial \Omega_{H}, \quad u_{0}^{0} \text { is outgoing. }
$$

For each $(i, k) \neq(0,0)$, the function $u_{i}^{k}$ only belongs to $H_{0, l o c}^{1}\left(\Omega_{H}\right)$ and satisfies

$$
\Delta u_{i}^{k}+\omega^{2} u_{i}^{k}=0 \text { in } \Omega_{H}, \quad \frac{\partial u_{i}^{k}}{\partial n}=0 \text { on } \partial \Omega_{H} \backslash\{\mathbf{0}\}, \quad u_{i}^{k} \text { is outgoing. }
$$

Moreover, the singularity at the origin of $u_{i}^{k}$ is limited "at order $i-k$ ", namely

$$
\mathcal{L}_{p}^{0}\left(u_{i}^{k}\right)=0, \quad \forall p>i-k
$$

Remark 2.2. As $f$ is compactly supported in $\Omega_{H}$ and due to equation (2.15), the functions $u_{i}^{k}$ 's belong to $\mathcal{V}\left(\Omega_{H}^{R}\right)$ and therefore are in the domain of $\mathcal{L}_{p}^{0}$ and $\mathcal{L}_{p}^{1}$, for $p \in \mathbb{N}$.

Remark 2.3. For convenience, we set

$$
u_{i}^{k} \equiv 0 \text { for }(i, k) \notin \mathbf{J} \text {. }
$$




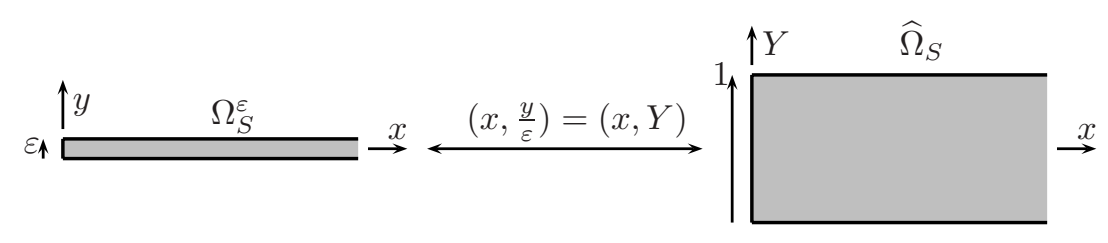

Figure 5. Slot-field domain.

Asymptotic expansion of the slot-field. This expansion holds in the slot-field domain $\Omega_{S}^{\varepsilon}$, see Figure 5 . In such a domain, it is natural to introduce the scaling $(x, Y)=(x, y / \varepsilon)$, so that

$$
\left.(x, y) \in \Omega_{S}^{\varepsilon} \Longleftrightarrow(x, y / \varepsilon) \in \widehat{\Omega}_{S}=\right] 0,+\infty[\times]-1 / 2,1 / 2[,
$$

where $\widehat{\Omega}_{S}$ is a normalized (or canonical) slot of width 1 . If we introduce the functions

$$
U^{\varepsilon}(x, Y)=u^{\varepsilon}(x, \varepsilon Y)
$$

we have

$$
U^{\varepsilon}(x, Y)=\sum_{i=0}^{+\infty} \sum_{k=0}^{i}\left(\frac{\omega \varepsilon}{2}\right)^{i} \log ^{k}\left(\frac{\omega \varepsilon}{2}\right) U_{i}^{k}(x)+o\left(\varepsilon^{\infty}\right), \quad(x, Y) \in \Omega_{S},
$$

where the functions $U_{i}^{k}$ 's solutions of the $1 \mathrm{D}$-Helmholtz equation defined on $\mathbb{R}^{+}$

$$
\frac{\mathrm{d}^{2} U_{i}^{k}}{\mathrm{~d} x^{2}}+\omega^{2} U_{i}^{k}=0
$$

Taking into account the outgoing condition, we have

$$
U_{i}^{k}(x)=U_{i}^{k}(0) \exp (\mathbf{i} \omega x)
$$

Remark 2.4. The reader will notice that the $y$-dependence of $u^{\varepsilon}$ is contained in the $o\left(\varepsilon^{\infty}\right)$ term.

Remark 2.5. By convention, we set

$$
U_{i}^{k} \equiv 0 \text { for }(i, k) \notin \mathbf{J} .
$$

The near-field expansion. This expansion will be valid in a small neighborhood of the origin. To define it properly we introduce the domain $\Omega_{N}^{\varepsilon}$ (it coincides with $\Omega_{\varepsilon}$ in the absence of obstacle, see Fig. 6)

$$
(x, y) \in \Omega_{N}^{\varepsilon} \quad \Longleftrightarrow \quad(x / \varepsilon, y / \varepsilon) \in \widehat{\Omega}_{N}=(]-\infty, 0[\times \mathbb{R}) \cup\left(\left[0,+\infty[\times]-\frac{1}{2}, \frac{1}{2}[) .\right.\right.
$$

The domain $\widehat{\Omega}_{N}$ is its normalized version. In [10], it was shown that there exists a family of functions $\mathcal{U}_{i}^{k} \in$ $H_{l o c}^{1}\left(\widehat{\Omega}_{N}\right)$ such that one has the formal asymptotic expansion

$$
\begin{gathered}
u^{\varepsilon}(x, y)=\sum_{i=0}^{+\infty} \sum_{k=0}^{i}\left(\frac{\omega \varepsilon}{2}\right)^{i} \log ^{k}\left(\frac{\omega \varepsilon}{2}\right) \mathcal{U}_{i}^{k}\left(\frac{x}{\varepsilon}, \frac{y}{\varepsilon}\right)+o\left(\varepsilon^{\infty}\right), \quad \text { for }\|(x, y)\|<R . \\
\mathcal{U}_{i}^{k} \equiv 0, \quad \text { with }(i, k) \notin \mathbf{J},
\end{gathered}
$$



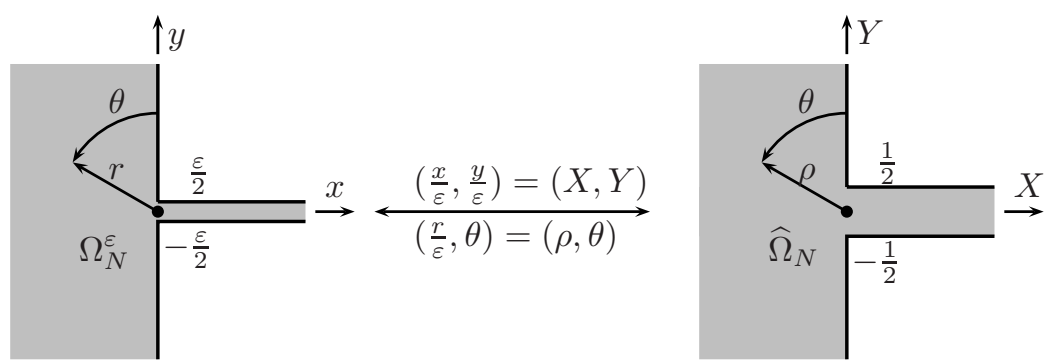

Figure 6. Near-field domain.

the function $\mathcal{U}_{i}^{k}$ satisfies the embedded Laplace equation

$$
\Delta \mathcal{U}_{i}^{k}=-4 \mathcal{U}_{i-2}^{k} \text { in } \widehat{\Omega}_{N} \quad \text { and } \quad \frac{\partial \mathcal{U}_{i}^{k}}{\partial n}=0 \text { on } \partial \widehat{\Omega}_{N} .
$$

Moreover, they are related to the far-fields through the following matching conditions

$$
\mathcal{U}_{i}^{k}(\rho, \theta)=\sum_{p=0}^{+\infty} \sum_{l=0}^{+\infty}\left[\left(\mathcal{L}_{p}^{1}\left(u_{i-p-l}^{k}\right)+\frac{2}{\pi} \mathcal{L}_{p}^{0}\left(u_{i-p-l}^{k-1}\right)\right) j_{p, l}(\rho, \theta)+\mathcal{L}_{p}^{0}\left(u_{i+p-l}^{k}\right) y_{p, l}(\rho, \theta)\right]
$$

for $\rho>\frac{1}{2}, \theta \in[0 ; \pi]$ where $(\rho, \theta)$ are the polar coordinates associated to $X$ and $Y$, and to the slot-fields via the matching conditions

$$
\mathcal{U}_{i}^{k}(X, Y)=\sum_{l=0}^{i-k} U_{i-l}^{k}(0) \frac{(2 \mathbf{i} X)^{l}}{l !}+\delta \mathcal{U}_{i}^{k}(X, Y), \quad \text { for } X>0,-\frac{1}{2}<Y<\frac{1}{2}
$$

with $\left|\delta \mathcal{U}_{i}^{k}(X, Y)\right| \leqslant \frac{C_{m}}{X^{m}}$

Remark 2.6. In the right hand side of (2.27), we have explicitly used the convention $u_{i}^{k} \equiv 0$ for $(i, k) \notin \mathbf{J}$. The convergence of this series is proved in [22].

The existence and uniqueness result. In [10], Theorem 4.1, we have proved the following result.

Theorem 2.1. There exists a unique family of functions $u_{i}^{k} \in H_{0, l o c}^{1}\left(\Omega_{H}\right), \mathcal{U}_{i}^{k} \in H_{l o c}^{1}\left(\widehat{\Omega}_{N}\right)$, and $U_{i}^{k} \in C^{\infty}\left(\mathbb{R}^{+}\right)$ with $(i, k) \in \mathbf{J}$ satisfying equations (2.14), (2.15), (2.16), (2.21), (2.26), (2.27), and (2.28). Moreover,

- the near-fields have the following asymptotic behaviours at infinity

$$
\begin{cases}\text { (i) } \mathcal{U}_{i}^{k}(X, Y) \leqslant C X^{i-k}, & \text { for } X>0 \\ \text { (ii) } \mathcal{U}_{i}^{k}(\rho, \theta) \leqslant C \rho^{i-k}, & \text { for } X<0\end{cases}
$$

- the far-fields satisfy

$$
\mathcal{L}_{p}^{0}\left(u_{i}^{k}\right)=0, \quad \text { for all } p \geqslant i-k
$$

\subsection{The main results}

In this section, we state three theorems which specify the sense to be given to the expansions (2.13), (2.19) and (2.24). Note that the proofs of these theorems are postponed to Section 3.

The following theorem concerns the far-field approximation. 
Theorem 2.2. For any compact set $F_{H} \subset \overline{\Omega_{H}} \backslash\{0\}$ and any $p \in \mathbb{N}$, there exists a positive constant $C_{p}=$ $C_{p}\left(\omega, F_{H}, \operatorname{supp}(f)\right)$ such that

$$
\left\|u^{\varepsilon}-\sum_{i=0}^{p} \sum_{k=0}^{i}\left(\frac{\omega \varepsilon}{2}\right)^{i} \log ^{k}\left(\frac{\omega \varepsilon}{2}\right) u_{i}^{k}\right\|_{H^{1}\left(F_{H}\right)} \leqslant C_{p}\left(\frac{\omega \varepsilon}{2}\right)^{p+1}\left|\log \left(\frac{\omega \varepsilon}{2}\right)\right|^{p}\|f\|_{L^{2}} .
$$

The second result concerns the slot-field approximation in scaled variables.

Theorem 2.3. With $U^{\varepsilon}$ defined by (2.18), for any compact set $F_{S} \subset\left\{(x, y) \in \overline{\widehat{\Omega}_{S}} / x \neq 0\right\}$ and any $p \in \mathbb{N}$, there exists a positive constant $C_{p}=C_{p}\left(\omega, F_{S}\right.$, $\left.\operatorname{supp}(f)\right)>0$ such that

$$
\left\|U^{\varepsilon}-\sum_{i=0}^{p} \sum_{k=0}^{i}\left(\frac{\omega \varepsilon}{2}\right)^{i} \log ^{k}\left(\frac{\omega \varepsilon}{2}\right) U_{i}^{k}\right\|_{H^{1}\left(F_{S}\right)} \leqslant C_{p}\left(\frac{\omega \varepsilon}{2}\right)^{p+1}\left|\log \left(\frac{\omega \varepsilon}{2}\right)\right|^{p+1}\|f\|_{L^{2}} .
$$

For the near-field approximation result, we first notice that for any compact $F_{N}$ of $\overline{\widehat{\Omega}_{N}}$ and for $\varepsilon$ small enough $\varepsilon F_{N}$ is included in $\Omega_{\varepsilon}$ and we can define

$$
\mathcal{U}^{\varepsilon}(X, Y)=u^{\varepsilon}(\varepsilon X, \varepsilon Y), \quad \forall(X, Y) \in F_{N} .
$$

Theorem 2.4. For any compact subset $F_{N}$ of $\overline{\widehat{\Omega}_{N}}$ and any $p \in \mathbb{N}$, there exists a positive constant $C_{p}=$ $C_{p}\left(\omega, F_{N}, \operatorname{supp}(f)\right)>0$ such that

$$
\left\|\mathcal{U}^{\varepsilon}-\sum_{i=0}^{p} \sum_{k=0}^{i}\left(\frac{\omega \varepsilon}{2}\right)^{i} \log ^{k}\left(\frac{\omega \varepsilon}{2}\right) \mathcal{U}_{i}^{k}\right\|_{H^{1}\left(F_{N}\right)} \leqslant C_{p}\left(\frac{\omega \varepsilon}{2}\right)^{p+1}\left|\log \left(\frac{\omega \varepsilon}{2}\right)\right|^{p+1}\|f\|_{L^{2}} .
$$

Remark 2.7. We cannot claim that the formal series (2.13), (2.19), and (2.24) converge, since the constants $C_{p}$ depend on $p$.

Remark 2.8. Due to the uniqueness of the generalized Taylor expansion, there exists a unique family of farfields $\left\{u_{i}^{k}\right\}_{(i, k) \in \mathbf{J}}$ - resp. slot-fields $\left\{U_{i}^{k}\right\}_{(i, k) \in \mathbf{J}}$ and near-fields $\left\{\mathcal{U}_{i}^{k}\right\}_{(i, k) \in \mathbf{J}}$ - satisfying inequalities (2.31) resp. (2.32) and (2.34) - for all $p \in \mathbb{N}$.

\section{ERROR ANALYSIS}

\subsection{A global approximation result}

A natural way for defining an approximation $\widetilde{u}_{n}^{\varepsilon}$ of $u^{\varepsilon}$ is to construct a function that coincides

(i) outside a small neighborhood of the origin and $x<0$, with the truncated far-field expansion $u_{n}^{\varepsilon}$

$$
u_{H, n}^{\varepsilon}(\mathbf{x}):=\sum_{i=0}^{n} \sum_{k=0}^{i}\left(\frac{\omega \varepsilon}{2}\right)^{i}\left(\log \frac{\omega \varepsilon}{2}\right)^{k} u_{i}^{k}(\mathbf{x}), \quad \text { in } \Omega_{H}
$$

(ii) inside a small neighborhood of the origin, with the truncated near-field expansion

$$
u_{N, n}^{\varepsilon}(\mathbf{x}):=\sum_{i=0}^{n} \sum_{k=0}^{i}\left(\frac{\omega \varepsilon}{2}\right)^{i}\left(\log \frac{\omega \varepsilon}{2}\right)^{k} \mathcal{U}_{i}^{k}\left(\frac{\mathbf{x}}{\varepsilon}\right), \quad \text { in } \Omega_{N}^{\varepsilon}
$$


(iii) inside the slot and $x$ "large" enough, with the truncated slot-field expansion $U_{n}^{\varepsilon}$

$$
u_{S, n}^{\varepsilon}(\mathbf{x}):=\sum_{i=0}^{n} \sum_{k=0}^{i}\left(\frac{\omega \varepsilon}{2}\right)^{i}\left(\log \frac{\omega \varepsilon}{2}\right)^{k} U_{i}^{k}(x), \quad \text { in } \Omega_{S}^{\varepsilon} ;
$$

(iv) a linear combination of these three fields in the intermediate zones.

This can be done via a partition of unity. To do so, we first introduce $\eta_{H}(\varepsilon)>0$ and $\eta_{S}(\varepsilon)>0$ for defining the size of a small neighborhood of the origin. These are a priori quantities that we authorize to vary with $\varepsilon$ in order to optimize the resulting error estimate. Obviously $\eta_{H}(\varepsilon)$ and $\eta_{S}(\varepsilon)$ are devoted to tend to zero with $\varepsilon$ to have a good approximation for the near field. However $\eta_{H}(\varepsilon)$ can not vanish too quickly because of the singularity of the far-fields $u_{i}^{k}$. This will clearly appear in the proof and will be more precise in (3.12). Let us introduce the domains

$$
\left\{\begin{array}{l}
B_{H}^{\varepsilon}=\left\{(r, \theta) \in \Omega_{H} \mid 0 \leqslant r \leqslant 2 \eta_{H}(\varepsilon)\right\}, \\
B_{S}^{\varepsilon}=\left\{(x, y) \in \Omega_{S} \mid 0 \leqslant x \leqslant 2 \eta_{S}(\varepsilon)\right\}, \\
B_{N}^{\varepsilon}=B_{H}^{\varepsilon} \cup B_{S}^{\varepsilon} .
\end{array}\right.
$$

Next, we introduce a 1D-cut-off function $\chi$

$$
\chi \in C^{\infty}\left(\mathbb{R}^{+} ; \mathbb{R}^{+}\right) \text {with } \chi(z)=0 \text { if } z \leq 1 \text { and } \chi(z)=1 \text { if } z \geq 2 .
$$

We define the function $\chi_{H}^{\varepsilon} \in C^{\infty}\left(\Omega_{\varepsilon}\right)$ such that

$$
\begin{cases}\chi_{H}^{\varepsilon}(\mathbf{x})=1, & \text { in } \Omega_{H} \backslash B_{H}^{\varepsilon}, \\ \chi_{H}^{\varepsilon}(\mathbf{x})=0, & \text { in } \Omega_{S}^{\varepsilon}, \\ \chi_{H}^{\varepsilon}(\mathbf{x})=\chi\left(|\mathbf{x}| / \eta_{H}(\varepsilon)\right), & \text { in } B_{H}^{\varepsilon} .\end{cases}
$$

Similarly, we introduce $\chi_{S}^{\varepsilon} \in C^{\infty}\left(\Omega_{\varepsilon}\right)$ such that

$$
\begin{cases}\chi_{S}^{\varepsilon}(x, y)=0, & \text { in } \Omega_{H}, \\ \chi_{S}^{\varepsilon}(x, y)=1, & \text { in } \Omega_{S}^{\varepsilon} \backslash B_{S}^{\varepsilon}, \\ \chi_{S}^{\varepsilon}(x, y)=\chi\left(x / \eta_{S}(\varepsilon)\right), & \text { in } B_{S}^{\varepsilon} .\end{cases}
$$

The reader will notice that by construction

$$
\frac{\partial \chi_{H}^{\varepsilon}}{\partial n}=\frac{\partial \chi_{S}^{\varepsilon}}{\partial n}=0 \quad \text { on } \partial \Omega_{\varepsilon} .
$$

Definition 3.1. The global approximation of order $n$ is the function $\tilde{u}_{n}^{\varepsilon}$ from $\Omega_{\varepsilon}$ to $\mathbb{C}$ defined by

$$
\tilde{u}_{n}^{\varepsilon}=\chi_{H}^{\varepsilon} u_{H, n}^{\varepsilon}+\chi_{S}^{\varepsilon} u_{S, n}^{\varepsilon}+\left(1-\chi_{H}^{\varepsilon}-\chi_{S}^{\varepsilon}\right) u_{N, n}^{\varepsilon} .
$$

By construction, one has

$$
\begin{cases}\widetilde{u}_{n}^{\varepsilon}=u_{H, n}^{\varepsilon}, & \text { in } \Omega_{H} \backslash B_{H}^{\varepsilon}, \\ \widetilde{u}_{n}^{\varepsilon}=u_{S, n}^{\varepsilon}, & \text { in } \Omega_{S}^{\varepsilon} \backslash B_{S}^{\varepsilon},\end{cases}
$$

and $\widetilde{u}_{n}^{\varepsilon}$ coincides with $u_{N, n}^{\varepsilon}$ in a neighborhood of the origin. The following theorem will be proved in Section 3.3 . 
Theorem 3.2. Assume that $\eta_{H}(\varepsilon)<R / 2$ with $R$ such that $(2.8)$ holds. For all bounded open $O \subset \mathbb{R}^{2}$, for all $m$ and $n$ in $\mathbb{N}$, and for all $f$ compactly supported in $\Omega_{H}$, there exist a real $C_{n}=C_{n}(O, m, f, \omega)>0$ and $\varepsilon_{0}>0$ satisfying for $\varepsilon<\varepsilon_{0}$ :

$$
\left\{\begin{aligned}
\left\|u^{\varepsilon}-\widetilde{u}_{n}^{\varepsilon}\right\|_{H^{1}\left(O \cap \Omega_{\varepsilon}\right)} \leqslant & C_{n}\left|\log \frac{\omega \varepsilon}{2}\right|^{n}\left|\log \frac{\omega \eta_{H}(\varepsilon)}{2}\right|^{3 / 2}\left[\left(\frac{\omega \eta_{H}(\varepsilon)}{2}\right)^{n+1}+\left(\frac{\varepsilon}{\eta_{H}(\varepsilon)}\right)^{n+1}\right] \\
& +C_{n}\left|\log \frac{\omega \varepsilon}{2}\right|^{n} \frac{\sqrt{\varepsilon}}{\eta_{S}(\varepsilon)}\left[\left(\frac{\omega \eta_{S}(\varepsilon)}{2}\right)^{n+1}+\left(\frac{\varepsilon}{\eta_{S}(\varepsilon)}\right)^{m}\right]
\end{aligned}\right.
$$

We see on estimate (3.11) that, in order to deduce a convergence result when $\varepsilon$ goes to zero, we need that the functions $\eta_{S}$ and $\eta_{H}$ satisfy the double property

$$
\lim _{\varepsilon \rightarrow 0} \eta(\varepsilon)=0 \text { and } \lim _{\varepsilon \rightarrow 0} \varepsilon / \eta(\varepsilon)=0 .
$$

To optimize the choice of $\eta_{H}(\varepsilon)$ and $\eta_{S}(\varepsilon)$ we first choose $\eta_{H}(\varepsilon)$ in order to minimize the quantity

$$
\left(\frac{\omega \eta_{H}(\varepsilon)}{2}\right)^{n+1}+\left(\frac{\varepsilon}{\eta_{H}(\varepsilon)}\right)^{n+1}
$$

which appear in the right hand side of (3.11). This leads to

$$
\frac{\omega \eta_{H}(\varepsilon)}{2}=\left(\frac{\omega \varepsilon}{2}\right)^{1 / 2}
$$

Next, it suffices to adjust the choice of $\eta_{S}(\varepsilon)$ and $m$ in such a way that the second term in (3.11) decays to zero with approximately the same speed. This is obtained by choosing

$$
\frac{\omega \eta_{S}(\varepsilon)}{2}=\left(\frac{\omega \varepsilon}{2}\right)^{1 / 2} \text { and } m=n+1 .
$$

Finally, applying Theorem 3.2 (note that $\eta_{H}(\varepsilon)<R / 2$ for $\varepsilon$ small enough), we have proved the following corollary.

Corollary 3.3. For any $n$ and any compact set $O \subset \mathbb{R}^{2}$, there exists a constant $C_{n}=C_{n}(O, f, \omega)$ such that:

$$
\left\|u^{\varepsilon}-\widetilde{u}_{n}^{\varepsilon}\right\|_{H^{1}\left(O \cap \Omega_{\varepsilon}\right)} \leqslant C_{n}\left|\log \frac{\omega \varepsilon}{2}\right|^{n+\frac{3}{2}}\left(\frac{\omega \varepsilon}{2}\right)^{\frac{n+1}{2}} .
$$

\subsection{Local error estimates: proof of Theorems 2.2, 2.3, and 2.4}

In this section, we show that the Theorems 2.2, 2.3, and 2.4 are corollaries of Theorem 3.2 (more precisely of estimate (3.16) of Cor. 3.3).

Proof of Theorem 2.2. Let $F_{H}$ be a compact set of $\Omega_{H} \backslash\{\mathbf{0}\}$. We choose $O=F_{H}$ and $n=2 p+2$ in Corollary 3.3 to obtain

$$
\left\|u^{\varepsilon}-\widetilde{u}_{2 p+3}^{\varepsilon}\right\|_{H^{1}\left(F_{H}\right)} \leqslant C_{p}\left|\log \frac{\omega \varepsilon}{2}\right|^{2 p+\frac{7}{2}}\left(\frac{\omega \varepsilon}{2}\right)^{p+\frac{3}{2}} \leqslant C_{p}\left|\log \frac{\omega \varepsilon}{2}\right|^{p}\left(\frac{\omega \varepsilon}{2}\right)^{p+1},
$$

where we have used $\left|\log \frac{\omega \varepsilon}{2}\right|^{p+\frac{7}{2}}\left(\frac{\omega \varepsilon}{2}\right)^{\frac{1}{2}} \leqslant C_{p}$ and where the $C_{p}$ 's denote generically the constants depending on $p$. Since $F_{H}$ does not include a small neighborhood of $\mathbf{0}$, one has for $\varepsilon$ small enough

$$
\widetilde{u}_{2 p+3}^{\varepsilon}=u_{H, 2 p+3}^{\varepsilon} \Rightarrow\left\|u^{\varepsilon}-u_{H, 2 p+3}^{\varepsilon}\right\|_{H^{1}\left(F_{H}\right)} \leqslant C_{p}\left|\log \frac{\omega \varepsilon}{2}\right|^{p}\left(\frac{\omega \varepsilon}{2}\right)^{p+1} .
$$


Moreover, we remark that (the $u_{i}^{k}$ 's do not depend on $\varepsilon$ )

$$
\left\|u_{H, 2 p+3}^{\varepsilon}-u_{H, p}^{\varepsilon}\right\|_{H^{1}\left(F_{H}\right)} \leqslant \sum_{i=p+1}^{2 p+3} \sum_{k=0}^{i-1}\left(\frac{\omega \varepsilon}{2}\right)^{i}\left|\log \frac{\omega \varepsilon}{2}\right|^{k}\left\|u_{i}^{k}\right\|_{H^{1}\left(F_{H}\right)} \leqslant C_{p}\left|\log \frac{\omega \varepsilon}{2}\right|^{p}\left(\frac{\omega \varepsilon}{2}\right)^{p+1} .
$$

The conclusion follows by triangular inequality from (3.18) and (3.19).

Proof of Theorem 2.4. Let $O \subset \mathbb{R}^{2}$ be an open subset containing the point $\mathbf{0}$. We choose $n=2 p+4$ and we apply Corollary 3.3

$$
\left\|u^{\varepsilon}-\widetilde{u}_{2 p+4}^{\varepsilon}\right\|_{H^{1}\left(O \cap \Omega_{\varepsilon}\right)} \leqslant C_{p}\left|\log \frac{\omega \varepsilon}{2}\right|^{2 p+\frac{11}{2}}\left(\frac{\omega \varepsilon}{2}\right)^{p+\frac{5}{2}} \leqslant C_{p}\left|\log \frac{\omega \varepsilon}{2}\right|^{p+1}\left(\frac{\omega \varepsilon}{2}\right)^{p+2} .
$$

Let $F_{N}$ be a compact set of the closure of $\widehat{\Omega}_{N}$. Since for $\varepsilon$ small enough, $\varepsilon F_{N} \subset O \cap \Omega_{\varepsilon}$, one has

$$
\left\|u^{\varepsilon}-\widetilde{u}_{2 p+4}^{\varepsilon}\right\|_{H^{1}\left(\varepsilon F_{N}\right)} \leqslant C_{p}\left|\log \frac{\omega \varepsilon}{2}\right|^{p+1}\left(\frac{\omega \varepsilon}{2}\right)^{p+2}
$$

Moreover, for $\varepsilon$ small enough, one has in $\varepsilon F_{N}$

$$
\widetilde{u}_{2 p+4}^{\varepsilon}(\mathbf{x})=u_{N, 2 p+4}^{\varepsilon}(\mathbf{x}) \quad \Longrightarrow \quad\left\|u^{\varepsilon}-u_{N, 2 p+4}^{\varepsilon}\right\|_{H^{1}\left(\varepsilon F_{N}\right)} \leqslant C_{p}\left|\log \frac{\omega \varepsilon}{2}\right|^{p+1}\left(\frac{\omega \varepsilon}{2}\right)^{p+2}
$$

With $\mathcal{U}^{\varepsilon}(\mathbf{X})=u^{\varepsilon}(\varepsilon \mathbf{X})$ and $\mathcal{U}_{N, n}^{\varepsilon}(\mathbf{X})=u_{N, n}^{\varepsilon}(\varepsilon \mathbf{X})$, scaling this equation $(\mathbf{x}=\varepsilon \mathbf{X})$

$$
\left\{\begin{array}{l}
\left\|\mathcal{U}^{\varepsilon}-\mathcal{U}_{N, 2 p+4}^{\varepsilon}\right\|_{L^{2}\left(F_{N}\right)}=\frac{1}{\varepsilon}\left\|u^{\varepsilon}-u_{N, 2 p+4}^{\varepsilon}\right\|_{L^{2}\left(\varepsilon F_{N}\right)} \\
\left\|\nabla \mathcal{U}^{\varepsilon}-\mathcal{U}_{N, 2 p+4}^{\varepsilon}\right\|_{L^{2}\left(F_{N}\right)}=\left\|\nabla u^{\varepsilon}-u_{N, 2 p+4}^{\varepsilon}\right\|_{L^{2}\left(\varepsilon F_{N}\right)}
\end{array}\right.
$$

leads to

$$
\left\|\mathcal{U}^{\varepsilon}-\mathcal{U}_{N, 2 p+4}^{\varepsilon}\right\|_{H^{1}\left(F_{N}\right)} \leqslant C_{p}\left|\log \frac{\omega \varepsilon}{2}\right|^{p+1}\left(\frac{\omega \varepsilon}{2}\right)^{p+1} .
$$

Since none of the $\mathcal{U}_{i}^{k}$ depends on $\varepsilon$

$$
\left\{\begin{aligned}
\left\|\mathcal{U}_{N, 2 p+4}^{\varepsilon}-\mathcal{U}_{N, p}^{\varepsilon}\right\|_{H^{1}\left(F_{N}\right)} & \leqslant \sum_{i=p+1}^{2 p+4} \sum_{k=0}^{i}\left(\frac{\omega \varepsilon}{2}\right)^{i}\left|\log \frac{\omega \varepsilon}{2}\right|^{k}\left\|\mathcal{U}_{i}^{k}\right\|_{H^{1}\left(F_{N}\right)} \\
& \leqslant C_{p}\left|\log \frac{\omega \varepsilon}{2}\right|^{p+1}\left(\frac{\omega \varepsilon}{2}\right)^{p+1}
\end{aligned}\right.
$$

We conclude by triangular inequality from (3.24) and (3.25).

Proof of Theorem 2.3. It combines the arguments of Theorems 2.2 and 2.4. The details are left to the reader.

\subsection{Proof of the global error estimate (Thm. 3.2)}

Reduction to a bounded domain. The forthcoming analysis, in particular the stability analysis, will use compactness results that require to work in spaces of functions defined in a bounded domain. That is why we need to characterize the restriction of the solution $u^{\varepsilon}$ of our problem to a bounded domain. This can de done, if the domain is chosen large enough, by exploiting the outgoing nature of the solution.

Let $A>0$ be chosen sufficiently large in order that

$$
\forall \mathbf{x} \in \operatorname{supp}(f) \cup \mathcal{B}, \quad|\mathbf{x}|<A
$$




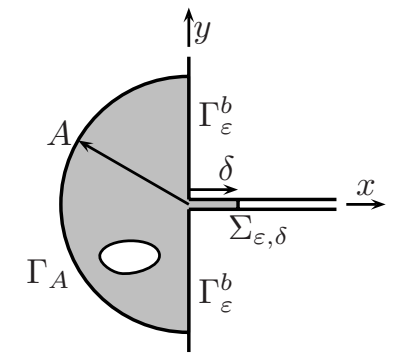

Figure 7. The domain $\Omega_{\varepsilon}^{b}$.

Let $\delta>0$. We define the bounded open set $\Omega_{\varepsilon}^{b}$, see Figure 7 , such that

$$
\overline{\Omega_{\varepsilon}^{b}}=\overline{\Omega_{H}^{A}} \cup \overline{\Omega_{S}^{\varepsilon, \delta}}
$$

where $\Omega_{H}^{A}$ and $\Omega_{S}^{\varepsilon, \delta}$ are defined by

$$
\left\{\begin{aligned}
\Omega_{H}^{A} & =\left\{\mathrm{x} \in \Omega_{H} /|\mathbf{x}|<A\right\} \\
\Omega_{S}^{\varepsilon, \delta} & =\left\{\mathrm{x} \in \Omega_{S}^{\varepsilon} / x<\delta\right\}
\end{aligned}\right.
$$

In our notation $b$ means "bounded" but also represents the couple $(A, \delta)$. The boundary of $\Omega_{\varepsilon}^{b}$ is split into three parts:

$$
\partial \Omega_{\varepsilon}^{b}=\Gamma_{A} \cup \Sigma_{\varepsilon, \delta} \cup \Gamma_{\varepsilon}^{b}
$$

where $\Gamma_{A}=\left\{\mathbf{x} \in \Omega_{H} /|\mathbf{x}|=A\right\}, \Sigma_{\varepsilon, \delta}=\left\{\mathbf{x} \in \Omega_{S}^{\varepsilon} / x=\delta\right\}$, and $\Gamma_{\varepsilon}^{b}=\partial \Omega_{\varepsilon}^{b} \cap \partial \Omega_{\varepsilon}$.

Let us point out that, in order to prove Theorem 3.2, we simply have to prove that the $H^{1}$-norm of $u^{\varepsilon}-\widetilde{u}_{n}^{\varepsilon}$ is bounded by the same expression as in right hand side of (3.11) where the constant $C_{n}$ depends on $A$ and $\delta$. Indeed, for any bounded open set $\mathcal{O}, \mathcal{O} \cap \Omega_{\varepsilon}$ is included in some $\Omega_{\varepsilon}^{b}$ for $A$ and $\delta$ appropriately chosen.

It is classical result that the restriction to $\Omega_{\varepsilon}^{b}$ of the solution $u^{\varepsilon}$ can be characterized as the unique solution (still denoted $u^{\varepsilon}$ for simplicity), of the boundary value problem

$$
\begin{cases}\Delta u^{\varepsilon}+\omega^{2} u^{\varepsilon}=-f, & \text { in } \Omega_{\varepsilon}^{b}, \\ \frac{\partial u^{\varepsilon}}{\partial n}=0, & \text { on } \Gamma_{\varepsilon}^{b}, \\ \frac{\partial u^{\varepsilon}}{\partial n}+T_{A} u^{\varepsilon}=0, & \text { on } \Gamma_{A}, \\ \frac{\partial u^{\varepsilon}}{\partial n}+T_{\varepsilon, \delta} u^{\varepsilon}=0, & \text { on } \Sigma_{\varepsilon, \delta}\end{cases}
$$

where $T_{A}$ and $T_{\varepsilon, \delta}$ are nonlocal boundary operators. More precisely:

(i) The operator $T_{\varepsilon, \delta} \in \mathcal{L}\left(H^{1 / 2}\left(\Sigma^{\varepsilon, \delta}\right) ; H^{-1 / 2}\left(\Sigma^{\varepsilon, \delta}\right)\right)$ is constructed such that the condition

$$
\frac{\partial u^{\varepsilon}}{\partial n}+T_{\varepsilon, \delta} u^{\varepsilon}=0, \quad \text { on } \Sigma_{\varepsilon, \delta}
$$

is a transparent (or exact) boundary condition for any outgoing solution $u$ of the Helmholtz equation in the semi-strip $\Omega_{S}^{\varepsilon}$. This operator is explicitly constructed, in diagonal form, by using the separation 
of variables in cartesian coordinates for the expression of such solutions. Introducing the orthonormal basis of $L^{2}\left(\Sigma_{\varepsilon, \delta}\right)\left(\Sigma_{\varepsilon, \delta}\right.$ is parametrized by $\left.y \in\right]-L / 2, L / 2[)$

$$
w_{0}^{\varepsilon}(y)=(1 / \varepsilon)^{\frac{1}{2}}, \quad w_{n}^{\varepsilon}(y)=(2 / \varepsilon)^{\frac{1}{2}} \cos \left[\frac{n \pi}{\varepsilon}\left(y+\frac{\varepsilon}{2}\right)\right], \quad \text { for } n \geq 1,
$$

the operator $T_{\varepsilon, \delta}$ is given for any $\varphi$ in $H^{1 / 2}\left(\Sigma_{\varepsilon, \delta}\right)$ by

$$
T_{\varepsilon, \delta} \varphi=-\mathbf{i} \omega \varphi_{0}^{\varepsilon, \delta} w_{0}^{\varepsilon}+\sum_{n=1}^{+\infty} \xi_{n}^{\varepsilon}(\omega) \varphi_{n}^{\varepsilon, \delta} w_{n}^{\varepsilon}, \quad \varphi_{n}^{\varepsilon, \delta}=\int_{-\frac{\varepsilon}{2}}^{\frac{\varepsilon}{2}} \varphi(\delta, y) w_{n}^{\varepsilon}(y) \mathrm{d} y
$$

where, assuming that $\varepsilon \omega<1$, we have for $n \in \mathbb{N}$

$$
\xi_{0}^{\varepsilon}(\omega)=-\mathbf{i} \omega, \quad \xi_{n}^{\varepsilon}(\omega)=\left(\frac{\pi^{2} n^{2}}{\varepsilon^{2}}-\omega^{2}\right)^{\frac{1}{2}}, \quad \forall n \in \mathbb{N}^{*} .
$$

As $\mathcal{R} e\left(\xi_{n}^{\varepsilon}(\omega)\right) \geqslant 0$ and $\mathcal{I} m\left(\xi_{n}^{\varepsilon}(\omega)\right) \leqslant 0, T_{\varepsilon, \delta}$ has the important properties

$$
\mathcal{R} e\left\langle T_{\varepsilon, \delta} \varphi ; \varphi\right\rangle_{\Sigma_{\varepsilon, \delta}} \geqslant 0 \text { and } \operatorname{Im}\left\langle T_{\varepsilon, \delta} \varphi ; \varphi\right\rangle_{\Sigma_{\varepsilon, \delta}} \leqslant 0
$$

where we use the notation $\langle\cdot, \cdot\rangle_{\Gamma}$ for the duality pairing between $H^{-\frac{1}{2}}(\Gamma)$ and $H^{\frac{1}{2}}(\Gamma)$.

(ii) The operator $T_{A} \in \mathcal{L}\left(H^{1 / 2}\left(\Gamma_{A}\right) ; H^{-1 / 2}\left(\Gamma_{A}\right)\right)$ is constructed such that the condition

$$
\frac{\partial u}{\partial n}+T_{A} u=0, \quad \text { on } \Gamma_{A}
$$

is a transparent (or exact) boundary condition for any outgoing solution $u$ of the Helmholtz equation in the half-space $\Omega_{H}$. Once again, this operator is explicitly constructed, in diagonal form, by using the separation of variables in polar coordinates for the expression of such solutions. We refer the reader to [9] for the analytic expression and only emphasize the important properties

$$
\mathcal{R} e\left\langle T_{A} \varphi ; \varphi\right\rangle_{\Gamma_{A}} \geqslant 0 \text { and } \operatorname{Im}\left\langle T_{A} \varphi ; \varphi\right\rangle_{\Gamma_{A}} \leqslant 0 .
$$

The error analysis. It is easy to show that the boundary value problem (3.30) is equivalent to the following variational problem

$$
\text { Find } u^{\varepsilon} \in H^{1}\left(\Omega_{\varepsilon}^{b}\right) \text { such that: } \quad a_{\varepsilon}^{b}\left(u^{\varepsilon}, v\right)=\int_{\Omega_{\varepsilon}^{b}} f \bar{v}, \quad \forall v \in H^{1}\left(\Omega_{\varepsilon}^{b}\right)
$$

where $a_{\varepsilon}^{b}: H^{1}\left(\Omega_{\varepsilon}^{b}\right) \times H^{1}\left(\Omega_{\varepsilon}^{b}\right) \longmapsto \mathbb{C}$ is the bilinear form given by

$$
a_{\varepsilon}^{b}(u ; v)=\int_{\Omega_{\varepsilon}^{b}}\left(\nabla u \nabla \bar{v}-\omega^{2} u \bar{v}\right)+\left\langle T_{\varepsilon, \delta} u, v\right\rangle_{\Sigma_{\varepsilon, \delta}}+\left\langle T_{A} u, v\right\rangle_{\Gamma_{A}} .
$$

Now, we associate an operator equation to this variational formulation

$$
\text { Find } u^{\varepsilon} \in H^{1}\left(\Omega_{\varepsilon}^{b}\right) \text { such that } \quad \mathbf{A}_{b}^{\varepsilon} u^{\varepsilon}=L_{b}^{\varepsilon},
$$

where $\mathbf{A}_{b}^{\varepsilon} \in \mathcal{L}\left(H^{1}\left(\Omega_{\varepsilon}^{b}\right)\right)$ is the continuous operator associated to $a_{\varepsilon}^{b}(\cdot, \cdot)$ via Riesz theorem

$$
\left(\mathbf{A}_{b}^{\varepsilon} u, v\right)_{H^{1}\left(\Omega_{\varepsilon}^{b}\right)}=a_{\varepsilon}^{b}(u, v), \quad \forall(u, v) \in H^{1}\left(\Omega_{\varepsilon}^{b}\right)^{2},
$$


and the vector $L_{b}^{\varepsilon} \in H^{1}\left(\Omega_{\varepsilon}^{b}\right)$ is defined via Riesz theorem

$$
\left(L_{b}^{\varepsilon}, v\right)_{H^{1}\left(\Omega_{\varepsilon}^{b}\right)}=\int_{\Omega_{\varepsilon}^{b}} f \bar{v}, \quad \forall v \in H^{1}\left(\Omega_{\varepsilon}^{b}\right) .
$$

From the well-posedness of (1.5), which is equivalent to (3.30), we deduce that $\mathbf{A}_{b}^{\varepsilon}$ is an isomorphism in $H^{1}\left(\Omega_{\varepsilon}^{b}\right)$. Subtracting $\mathbf{A}_{b}^{\varepsilon} \widetilde{u}_{n}^{\varepsilon}$ to the two members of equation, we get the following equation for the error we wish to estimate

$$
\mathbf{A}_{b}^{\varepsilon}\left(\widetilde{u}_{n}^{\varepsilon}-u^{\varepsilon}\right)=\mathbf{A}_{b}^{\varepsilon} \widetilde{u}_{n}^{\varepsilon}-L_{b}^{\varepsilon} .
$$

Therefore, the error analysis relies on two fundamental results which will be proven in the next two sections

(i) a stability result (Lem. 3.4) which provides an upper bound for the norm of the solution operator $\left(\mathbf{A}_{b}^{\varepsilon}\right)^{-1}$

Lemma 3.4 (stability). There exists a positive constant $C=C(\omega, A, \delta)$ (independent of $\varepsilon$ ) such that

$$
\left\|\left(\mathbf{A}_{b}^{\varepsilon}\right)^{-1}\right\|_{\mathcal{L}\left(H^{1}\left(\Omega_{\varepsilon}^{b}\right)\right)} \leqslant C
$$

(ii) a consistency estimate (Lem. 3.5) which provides an estimate of the residue $\mathbf{A}_{b}^{\varepsilon} \widetilde{u}_{n}^{\varepsilon}-L_{b}^{\varepsilon}$

Lemma 3.5 (consistency). For any $n>0$ and $m>0$, there exists a constant $C_{n, m}=C_{n, m}(\omega, R, \delta, f)$ (independent of $\varepsilon$ ) such that

$$
\left\{\begin{aligned}
\left\|\mathbf{A}_{b}^{\varepsilon} \widetilde{u}_{n}^{\varepsilon}-L_{b}^{\varepsilon}\right\|_{H^{1}\left(\Omega_{\varepsilon}^{b}\right)} \leqslant & C_{n, m}\left|\log \frac{\omega \varepsilon}{2}\right|^{n}\left|\log \frac{\omega \eta_{H}}{2}\right|^{\frac{3}{2}}\left[\left(\frac{\omega \eta_{H}}{2}\right)^{n+1}+\left(\frac{\varepsilon}{\eta_{H}}\right)^{n+1}\right] \\
& +C_{n, m}\left|\log \frac{\omega \varepsilon}{2}\right|^{n}\left(\frac{\sqrt{\varepsilon}}{\eta_{S}(\varepsilon)}\right)\left[\left(\frac{\omega \eta_{S}}{2}\right)^{n+1}+\left(\frac{\varepsilon}{\eta_{S}}\right)^{m}\right] .
\end{aligned}\right.
$$

Finally, the proof of Theorem 3.2 follows from the inequality (deduced from (3.43))

$$
\left\|\widetilde{u}_{n}^{\varepsilon}-u^{\varepsilon}\right\|_{H^{1}\left(\Omega_{\varepsilon}^{b}\right)} \leqslant\left\|\left(\mathbf{A}_{b}^{\varepsilon}\right)^{-1}\right\|_{\mathcal{L}\left(H^{1}\left(\Omega_{\varepsilon}^{b}\right)\right)}\left(\left\|\mathbf{A}_{b}^{\varepsilon} \widetilde{u}_{n}^{\varepsilon}-L_{b}^{\varepsilon}\right\|_{H^{1}\left(\Omega_{\varepsilon}^{b}\right)}\right) .
$$

\subsection{The stability result: proof of the Lemma 3.4}

We make a proof by contradiction. If $\left(\mathbf{A}_{b}^{\varepsilon}\right)^{-1}$ is not bounded independently of $\varepsilon$, then for $\varepsilon$ tending to zero, there exists a family of functions $\left\{u^{\varepsilon}\right\}$ parametrized by $\varepsilon$ such that

$$
\left\|u^{\varepsilon}\right\|_{H^{1}\left(\Omega_{\varepsilon}^{b}\right)}=1 \quad \text { and } \quad\left\|\mathbf{A}_{b}^{\varepsilon} u^{\varepsilon}\right\|_{H^{1}\left(\Omega_{\varepsilon}^{b}\right)} \rightarrow 0 \quad \text { for } \varepsilon \rightarrow 0 .
$$

Next, we decompose the proof into three steps.

Step 1. We first show that $u^{\varepsilon}$ does converge to zero weakly in $H^{1}\left(\Omega_{H}^{A}\right)$ so that, by compactness

$$
\lim _{\varepsilon \rightarrow 0}\left\|u^{\varepsilon}\right\|_{L^{2}\left(\Omega_{H}^{A}\right)}=0 .
$$

Indeed, as $\Omega_{H}^{A} \subset \Omega_{\varepsilon}^{b}$, one has

$$
\left\|u^{\varepsilon}\right\|_{H^{1}\left(\Omega_{H}^{A}\right)} \leqslant\left\|u^{\varepsilon}\right\|_{H^{1}\left(\Omega_{\varepsilon}^{b}\right)} .
$$

Hence, one can extract from $u^{\varepsilon}$ a family which converges weakly in $H^{1}\left(\Omega_{H}^{A}\right)$ to $u \in H^{1}\left(\Omega_{H}^{A}\right)$. Let us introduce the space of test functions $H_{00}^{1}\left(\Omega_{H}^{A}\right)$ defined by

$$
H_{00}^{1}\left(\Omega_{H}^{A}\right)=\left\{v \in H^{1}\left(\Omega_{H}^{A}\right) / \exists \varepsilon_{0}(v)>0, v(\mathbf{x})=0 \text { for }|\mathbf{x}|<\varepsilon_{0}\right\} .
$$


For all $v \in H_{00}^{1}\left(\Omega_{H}^{R}\right)$ and for $\varepsilon<\varepsilon_{0}(v)$, one has (use (3.39), (3.41) and the property of $v$ )

$$
\left(\mathbf{A}_{b}^{\varepsilon} u^{\varepsilon} ; v\right)_{H^{1}\left(\Omega_{\varepsilon}^{b}\right)}=\int_{\Omega_{H}^{A}}\left(\nabla u^{\varepsilon} \nabla \bar{v}-\omega^{2} u^{\varepsilon} \bar{v}\right)+\left\langle T_{A} u^{\varepsilon}, v\right\rangle_{\Gamma_{A}} .
$$

When $\varepsilon$ tends to 0 , this leads to

$$
\int_{\Omega_{H}^{A}}\left(\nabla u \nabla \bar{v}-\omega^{2} u \bar{v}\right)+\left\langle T_{A} u, v\right\rangle_{\Gamma_{A}}=0, \quad \forall v \in H_{00}^{1}\left(\Omega_{H}^{A}\right) .
$$

Thus, by density of $H_{00}^{1}\left(\Omega_{H}^{A}\right)$ in $H^{1}\left(\Omega_{H}^{A}\right)$, see [22], $u$ solves the variational problem

$$
\int_{\Omega_{H}^{A}}\left(\nabla u \nabla \bar{v}-\omega^{2} u \bar{v}\right)+\left\langle T_{A} u, v\right\rangle_{\Gamma_{A}}=0, \quad \forall v \in H^{1}\left(\Omega_{H}^{A}\right) .
$$

This means that $u$ is the restriction to $\Omega_{H}^{A}$ of the outgoing solution, still denoted $u$ for simplicity, of the boundary value problem in the domain $\Omega_{H}$ :

$$
\begin{cases}\Delta u+\omega^{2} u=0, & \text { in } \Omega_{H} \\ \frac{\partial u}{\partial n}=0, & \text { on } \partial \Omega_{H}\end{cases}
$$

This problem only admits the trivial solution as outgoing solution. Hence $u \equiv 0$.

Step 2. In $\Omega_{S}^{\varepsilon, \delta}$, we prove that

$$
\lim _{\varepsilon \rightarrow 0}\left\|u^{\varepsilon}\right\|_{L^{2}\left(\Omega_{S}^{\varepsilon, \delta}\right)}=0
$$

The proof is based on the orthogonal decomposition

$$
u^{\varepsilon}=P_{\varepsilon} u^{\varepsilon}+\left(u^{\varepsilon}-P_{\varepsilon} u^{\varepsilon}\right)
$$

where $P_{\varepsilon}$ is the orthogonal projector in $L^{2}$ onto $y$-independent functions

$$
\forall v \in H^{1}, \quad P_{\varepsilon} v(x, y)=P_{\varepsilon} v(x)=\frac{1}{\varepsilon} \int_{-\varepsilon / 2}^{\varepsilon / 2} v\left(x, y^{\prime}\right) \mathrm{d} y^{\prime} .
$$

Using for instance the expansion on the basis $w_{n}^{\varepsilon}$ (see (3.32)), one easily proves the inequality

$$
\forall v \in H^{1}\left(\Omega_{S}^{\varepsilon, \delta}\right), \quad\left\|v-P_{\varepsilon} v\right\|_{L^{2}\left(\Omega_{S}^{\varepsilon, \delta}\right)} \leq \frac{\varepsilon}{\sqrt{1+\pi^{2}}}\left\|u^{\varepsilon}\right\|_{H^{1}\left(\Omega_{S}^{\varepsilon, \delta}\right)} .
$$

Using Pythagoras theorem and (3.58), we have

$$
\left\|u^{\varepsilon}\right\|_{L^{2}\left(\Omega_{S}^{\varepsilon, \delta}\right)}^{2}=\left\|u^{\varepsilon}-P_{\varepsilon} u^{\varepsilon}\right\|_{L^{2}\left(\Omega_{S}^{\varepsilon, \delta}\right)}^{2}+\left\|P_{\varepsilon} u^{\varepsilon}\right\|_{L^{2}\left(\Omega_{S}^{\varepsilon, \delta}\right)}^{2} \leq\left\|P_{\varepsilon} u^{\varepsilon}\right\|_{L^{2}\left(\Omega_{S}^{\varepsilon, \delta}\right)}^{2}+C \varepsilon^{2} .
$$

On the other hand, we remark that

$$
\left\|P_{\varepsilon} u^{\varepsilon}\right\|_{L^{2}\left(\Omega_{S}^{\varepsilon, \delta}\right)}^{2}=\left\|U^{\varepsilon}\right\|_{L^{2}(0, \delta)}^{2}, \quad \text { with } \quad U^{\varepsilon}(x)=\frac{1}{\sqrt{\varepsilon}} \int_{-\varepsilon / 2}^{\varepsilon / 2} u^{\varepsilon}(x, y) \mathrm{d} y .
$$


Therefore, to prove (3.55), one has just to show that

$$
\lim _{\varepsilon \rightarrow 0}\left\|U^{\varepsilon}\right\|_{L^{2}(0, \delta)}=0
$$

Since $P_{\varepsilon}$ is a projection, we get

$$
\left\|U^{\varepsilon}\right\|_{H^{1}\left(\Omega_{S}^{\varepsilon, \delta}\right)} \leqslant\left\|P_{\varepsilon} u^{\varepsilon}\right\|_{H^{1}\left(\Omega_{S}^{\varepsilon, \delta}\right)} \leqslant\left\|u^{\varepsilon}\right\|_{L^{2}\left(\Omega_{S}^{\varepsilon, \delta}\right)} \leqslant\left\|u^{\varepsilon}\right\|_{H^{1}\left(\Omega_{S}^{\varepsilon, \delta}\right)} \leqslant\left\|u^{\varepsilon}\right\|_{H^{1}\left(\Omega_{\varepsilon}^{b}\right)}=1 .
$$

Moreover, since $\partial / \partial x$ and $P_{\varepsilon}$ commute, we have

$$
\left\|\frac{\mathrm{d} U^{\varepsilon}}{\mathrm{d} x}\right\|_{L^{2}(0, \delta)}=\left\|P_{\varepsilon} \frac{\partial u^{\varepsilon}}{\partial x}\right\|_{L^{2}\left(\Omega_{S}^{\varepsilon, \delta}\right)} \leqslant\left\|\frac{\partial u^{\varepsilon}}{\partial x}\right\|_{L^{2}\left(\Omega_{S}^{\varepsilon, \delta}\right)} \leqslant\left\|u^{\varepsilon}\right\|_{H^{1}\left(\Omega_{\varepsilon}^{b}\right)}=1 .
$$

By (3.62) and (3.63), $U^{\varepsilon}$ is bounded in $H^{1}(0, \delta)$. We can extract from $U^{\varepsilon}$ a subsequence with $\varepsilon \rightarrow 0$ which weakly converges to $U$ in $H^{1}(0, \delta)$ for $\varepsilon \rightarrow 0$. We prove below that

$$
\begin{aligned}
& \left\{\begin{array}{l}
\left.\frac{\mathrm{d}^{2} U}{\mathrm{~d} x^{2}}+\omega^{2} U=0, \quad \text { in }\right] 0, \delta[ \\
\frac{\mathrm{d} U}{\mathrm{~d} x}(\delta)-\mathbf{i} \omega U(\delta)=0
\end{array}\right. \\
& U(0)=0 .
\end{aligned}
$$

As the system (3.64), (3.65) only admits the trivial solution, we conclude that $U \equiv 0$. Thus, $U^{\varepsilon}$ does weakly converge to 0 in $H^{1}(0, \delta)$, and strongly in $L^{2}(0, \delta)$ by compactness. This proves (3.61) and thus (3.55).

To show (3.64), let $\mathbf{V}=\left\{\varphi \in H^{1}(0, \delta) / \varphi(0)=0\right\}$. If $\varphi \in \mathbf{V}$, its extension by 0 for $x \leq 0$, that we denote $\widetilde{\varphi}$, belongs to $H^{1}(-\infty, \delta)$, so that the test function $v$ defined in $\Omega_{\varepsilon}^{b}$ by

$$
v^{\varepsilon}(x, y)=\widetilde{\varphi}(x) w_{0}^{\varepsilon}(y)=\frac{1}{\sqrt{\varepsilon}} \widetilde{\varphi}(x)
$$

belongs to $H^{1}\left(\Omega_{\varepsilon}^{b}\right)$. Moreover, $v^{\varepsilon}$ is bounded independently of $\varepsilon$

$$
\left\|v^{\varepsilon}\right\|_{H^{1}\left(\Omega_{\varepsilon}^{b}\right)}^{2}=\|\varphi\|_{H^{1}(0, \delta)}^{2} .
$$

By definition of $\mathbf{A}_{b}^{\varepsilon}$, one has

$$
\left(\mathbf{A}_{b}^{\varepsilon} u^{\varepsilon} ; v^{\varepsilon}\right)_{H^{1}\left(\Omega_{\varepsilon}^{b}\right)}=\int_{0}^{\delta} \frac{\mathrm{d} U^{\varepsilon}}{\mathrm{d} x}(x) \overline{\frac{\mathrm{d} \varphi}{\mathrm{d} x}(x)}-\omega^{2} U^{\varepsilon}(x) \overline{\varphi(x)} \mathrm{d} x-\mathbf{i} \omega U^{\varepsilon}(\delta) \overline{\varphi(\delta)} .
$$

By Cauchy-Schwarz inequality, we have

$$
\left(\mathbf{A}_{b}^{\varepsilon} u^{\varepsilon} ; v^{\varepsilon}\right)_{H^{1}\left(\Omega_{\varepsilon}^{b}\right)} \leqslant\left\|\mathbf{A}_{b}^{\varepsilon} u^{\varepsilon}\right\|_{H^{1}\left(\Omega_{\varepsilon}^{b}\right)}\left\|v^{\varepsilon}\right\|_{H^{1}\left(\Omega_{\varepsilon}^{b}\right)} \rightarrow 0 \quad(\varepsilon \rightarrow 0),
$$

thanks to (3.47), and (3.67). By weak convergence of $U^{\varepsilon}$ in $H^{1}(0, \delta)$, taking the limit of (3.68) when $\varepsilon$ goes to 0 , we get

$$
\int_{0}^{\delta} \frac{\mathrm{d} U}{\mathrm{~d} x}(x) \frac{\overline{\mathrm{d} \varphi}}{\mathrm{d} x}(x)-\omega^{2} U(x) \overline{\varphi(x)} \mathrm{d} x-\mathbf{i} \omega U(\delta) \overline{\varphi(\delta)}=0, \quad \forall \varphi \in \mathbf{V} .
$$

This leads to (3.64).

To prove (3.65), we remark that, by weak convergence of $U^{\varepsilon}$ in $H^{1}(0, \delta)$, we have

$$
U(0)=\lim _{\varepsilon \rightarrow 0} U^{\varepsilon}(0)
$$


By definition of $U^{\varepsilon}$, we have

$$
U^{\varepsilon}(0)=\frac{1}{\sqrt{\varepsilon}} \int_{-\varepsilon / 2}^{\varepsilon / 2} u^{\varepsilon}(0, y) \mathrm{d} y=:\left(u^{\varepsilon}\right)_{0}^{\varepsilon}
$$

In [9], Lemma 4.1, we proved that (with $C$ independent of $\varepsilon$ )

$$
\left|\left(u^{\varepsilon}\right)_{0}^{\varepsilon}\right| \leqslant C \sqrt{\varepsilon|\log \varepsilon|}\left\|u^{\varepsilon}\right\|_{H^{1}\left(\Omega_{H}^{A}\right)} .
$$

Thanks to (3.47), we deduce (3.65) from (3.71) and (3.73).

Step 3. To get a contradiction, we compute $\left(\mathbf{A}_{b}^{\varepsilon} u^{\varepsilon} ; u^{\varepsilon}\right)_{H^{1}\left(\Omega_{\varepsilon}^{b}\right)}$

$$
\left(A_{b}^{\varepsilon} u^{\varepsilon} ; u^{\varepsilon}\right)_{H^{1}\left(\Omega_{\varepsilon}^{b}\right)}=\int_{\Omega_{\varepsilon}^{b}}\left(\left|\nabla u^{\varepsilon}\right|^{2}-\omega^{2}\left|u^{\varepsilon}\right|^{2}\right)+\left\langle T_{A} u^{\varepsilon}, u^{\varepsilon}\right\rangle_{\Gamma_{A}}+\left\langle T_{\varepsilon, \delta} u^{\varepsilon} ; u^{\varepsilon}\right\rangle_{\Sigma_{\varepsilon, \delta},}
$$

Due to properties (3.35) and (3.37) of $T_{A}$ and $T_{\varepsilon, \delta}$, taking the real value of (3.74), one has

$$
\int_{\Omega_{\varepsilon}^{b}}\left|\nabla u^{\varepsilon}\right|^{2} \leqslant \omega^{2} \int_{\Omega_{\varepsilon}^{b}}\left|u^{\varepsilon}\right|^{2}+\left|\mathcal{R} e\left(\mathbf{A}_{b}^{\varepsilon} u^{\varepsilon}, u^{\varepsilon}\right)_{H^{1}\left(\Omega_{\varepsilon}^{b}\right)}\right| .
$$

It follows from Cauchy-Schwarz inequality and from (3.47)

$$
\left|\mathcal{R} e\left(\mathbf{A}_{b}^{\varepsilon} u^{\varepsilon}, u^{\varepsilon}\right)_{H^{1}\left(\Omega_{\varepsilon}^{b}\right)}\right| \leqslant\left\|\mathbf{A}_{b}^{\varepsilon} u^{\varepsilon}\right\|_{H^{1}\left(\Omega_{\varepsilon}^{b}\right)}\left\|u^{\varepsilon}\right\|_{H^{1}\left(\Omega_{\varepsilon}^{b}\right)} \underset{\varepsilon \rightarrow 0}{\longrightarrow} 0
$$

Moreover, (3.48) and (3.55) imply $\lim _{\varepsilon \rightarrow 0}\left\|u^{\varepsilon}\right\|_{L^{2}\left(\Omega_{\varepsilon}^{b}\right)}=0$. Hence, from (3.75), we deduce that

$$
\lim _{\varepsilon \rightarrow 0}\left\|\nabla u^{\varepsilon}\right\|_{L^{2}\left(\Omega_{\varepsilon}^{b}\right)}=0 \quad \Longrightarrow \quad \lim _{\varepsilon \rightarrow 0}\left\|u^{\varepsilon}\right\|_{H^{1}\left(\Omega_{\varepsilon}^{b}\right)}=0
$$

This contradicts (3.47).

\subsection{The consistency result: proof of Lemma 3.5}

\subsubsection{Matching error between far-field and near-field}

We denote by $\mathcal{E}_{n}^{\varepsilon}$, the matching error of order $n$ between the far-field and the near-field, namely the function defined in the domain

by

$$
C_{H}^{\varepsilon}=\left\{(r, \theta) \in \Omega_{H} \mid \eta_{H}(\varepsilon) \leqslant r \leqslant 2 \eta_{H}(\varepsilon)\right\}
$$

$$
\mathcal{E}_{n}^{\varepsilon}=u_{N, n}^{\varepsilon}-u_{H, n}^{\varepsilon}
$$

Using polar coordinates, we have

$$
\mathcal{E}_{n}^{\varepsilon}(r, \theta)=\sum_{i=0}^{n} \sum_{k=0}^{i}\left(\frac{\omega \varepsilon}{2}\right)^{i}\left(\log \frac{\omega \varepsilon}{2}\right)^{k} \mathcal{U}_{i}^{k}\left(\frac{r}{\varepsilon}, \theta\right)-\sum_{i=0}^{n} \sum_{k=0}^{i}\left(\frac{\omega \varepsilon}{2}\right)^{i}\left(\log \frac{\omega \varepsilon}{2}\right)^{k} u_{i}^{k}(r, \theta)
$$

As its name suggests, the function $\mathcal{E}_{n}^{\varepsilon}$ measures the lack of matching between the truncated near-field expansion $\mathcal{U}_{n}^{\varepsilon}$ and the truncated far-field expansion $u_{n}^{\varepsilon}$. In fact by construction of the matching condition, see [10], the non truncated expansion corresponding to $n=+\infty$ matched up to $o\left(\varepsilon^{\infty}\right)$. The fact that $u_{n}^{\varepsilon}$ and $\mathcal{U}_{n}^{\varepsilon}$ fails to match is due to the truncation. 

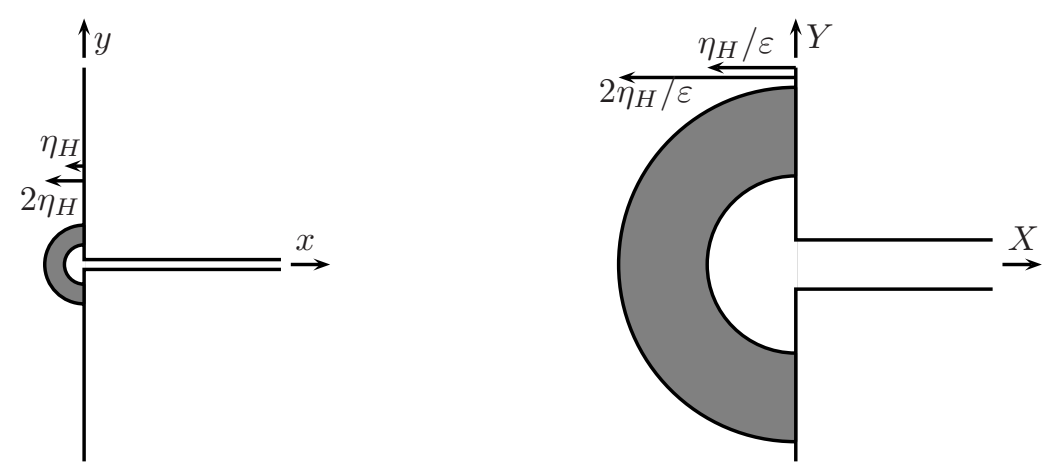

FiguRE 8. The intermediate zone between far-field and near-field.

As it will be proved in Lemma 3.6, this quantity is small in the transition zone, see Figure 8,

$$
(r, \theta) \in\left[\eta_{H}(\varepsilon), 2 \eta_{H}(\varepsilon)\right] \times[0 ; \pi] \Longleftrightarrow(\rho, \theta) \in\left[\frac{\eta_{H}}{\varepsilon} ; 2 \frac{\eta_{H}(\varepsilon)}{\varepsilon}\right] \times[0 ; \pi],
$$

which tends to zero in the physical coordinates and to infinity in the near-field coordinates, see (3.12).

Lemma 3.6. For any $n \in \mathbb{N}$, there exist a real $C(\omega, n, f)$ and $\varepsilon_{0}>0$ such that, for $\varepsilon<\varepsilon_{0}$,

$$
\left\{\begin{array}{l}
\left\|\mathcal{E}_{n}^{\varepsilon}\right\|_{L^{\infty}\left(C_{H}^{\varepsilon}\right)} \leqslant C\left|\log \frac{\omega \varepsilon}{2}\right|^{n}\left|\log \frac{\omega \eta_{H}}{2}\right|\left[\left(\frac{\varepsilon}{\eta_{H}}\right)^{n+1}+\left(\frac{\omega \eta_{H}}{2}\right)^{n+1}\right], \\
\left\|\nabla \mathcal{E}_{n}^{\varepsilon}\right\|_{L^{\infty}\left(C_{H}^{\varepsilon}\right)} \leqslant C\left|\log \frac{\omega \varepsilon}{2}\right|^{n}\left|\log \frac{\omega \eta_{H}}{2}\right| \frac{1}{\eta_{H}}\left[\left(\frac{\varepsilon}{\eta_{H}}\right)^{n+1}+\left(\frac{\omega \eta_{H}}{2}\right)^{n+1}\right] .
\end{array}\right.
$$

Proof. Here, we prove the estimate of the first line of (3.82). The same technique can be applied to the second line.

In what follows, we will skip the question of convergence of the series. The details, can be found in [22].

Let $n$ be a fixed integer and consider $(i, k) \in \mathbf{J}$ with $i \leqslant n$. Let us recall that

$$
u_{i}^{k}(r, \theta)=\sum_{p, l}\left(\mathcal{L}_{p}^{0}\left(u_{i}^{k}\right) y_{p, l}\left(\frac{\omega r}{2}, \theta\right)+\mathcal{L}_{p}^{1}\left(u_{i}^{k}\right) j_{p, l}\left(\frac{\omega r}{2}, \theta\right)\right)
$$

where by convention $\sum_{p, l}$ denotes $\sum_{p=0}^{+\infty} \sum_{l=0}^{+\infty}$.

In the transition zone, the argument $r$ is supposed to be small. We are going to separate in the series above the terms that decay faster than $r^{M}$. To do this, we observe that

$$
\begin{cases}j_{p, l}(z) \leqslant C_{p, l} z^{M+1}, & \text { if } p+l \geqslant M+1 \\ y_{p, l}(z) \leqslant C_{p, l} z^{M+1} \log z, & \text { if }-p+l \geqslant M+1 .\end{cases}
$$

Thus, we write

$$
\left\{\begin{aligned}
u_{i}^{k}(r, \theta)= & \sum_{l-p \leqslant M}\left[\mathcal{L}_{p}^{0}\left(u_{i}^{k}\right) y_{p, l}\left(\frac{\omega r}{2}, \theta\right)\right]+\sum_{p+l \leqslant M}\left[\mathcal{L}_{p}^{1}\left(u_{i}^{k}\right) j_{p, l}\left(\frac{\omega r}{2}, \theta\right)\right] \\
& +\sum_{l-p \geqslant M+1}\left[\mathcal{L}_{p}^{0}\left(u_{i}^{k}\right) y_{p, l}\left(\frac{\omega r}{2}, \theta\right)\right]+\sum_{p+l \geqslant M+1}\left[\mathcal{L}_{p}^{1}\left(u_{i}^{k}\right) j_{p, l}\left(\frac{\omega r}{2}, \theta\right)\right] .
\end{aligned}\right.
$$


Each term of the series in the second line of (3.85) is bounded by $C_{p, l}(\omega r / 2)^{M+1} \log (\omega r / 2)$. Checking carefully the convergence of these series — we omit this tedious detail — one proves that they are bounded by $C(\omega r / 2)^{M+1} \log (\omega r / 2)$.

Next, we choose $M=n-i$, so that

$$
u_{i}^{k}(r, \theta)=\sum_{l-p \leqslant n-i}\left[\mathcal{L}_{p}^{0}\left(u_{i}^{k}\right) y_{p, l}\left(\frac{\omega r}{2}, \theta\right)\right]+\sum_{p+l \leqslant n-i}\left[\mathcal{L}_{p}^{1}\left(u_{i}^{k}\right) j_{p, l}\left(\frac{\omega r}{2}, \theta\right)\right]+\delta u_{i}^{k}(r, \theta)
$$

where

$$
\left|\delta u_{i}^{k}(r, \theta)\right| \leqslant C\left(\frac{\omega \eta_{H}}{2}\right)^{n-i+1}\left|\log \left(\frac{\omega \eta_{H}}{2}\right)\right|, \quad \forall r \leqslant 2 \eta_{H}(\varepsilon) .
$$

On the other hand, we have (this is nothing but the matching condition (2.27))

$$
\mathcal{U}_{i}^{k}(\rho, \theta)=\sum_{p, l}\left[\left(\mathcal{L}_{p}^{1}\left(u_{i-p-l}^{k}\right)+\frac{2}{\pi} \mathcal{L}_{p}^{0}\left(u_{i-p-l}^{k-1}\right)\right) j_{p, l}(\rho, \theta)+\mathcal{L}_{p}^{0}\left(u_{i+p-l}^{k}\right) y_{p, l}(\rho, \theta)\right]
$$

In the expression (3.79) the argument $\rho$ is equal to $\frac{r}{\varepsilon}$ which is large in the transition zone. That is why in the sum (3.88) we separate the terms that decay faster than $\rho^{-M}$ from the others. The only terms of this type are provided by the $y_{p, l}$ for $l-p \leqslant-M-1$. Indeed

$$
y_{p, l}(z, \theta) \leqslant C z^{-M-1} \quad \text { for } l-p \leqslant-M-1 .
$$

As a consequence if we write

$$
\left\{\begin{aligned}
\mathcal{U}_{i}^{k}(\rho, \theta)= & \sum_{l-p \geqslant-M}\left[\mathcal{L}_{p}^{0}\left(u_{i+p-l}^{k}\right) y_{p, l}(\rho, \theta)\right]+\sum_{p, l}\left[\left(\mathcal{L}_{p}^{1}\left(u_{i-p-l}^{k}\right)+\frac{2}{\pi} \mathcal{L}_{p}^{0}\left(u_{i-p-l}^{k-1}\right)\right) j_{p, l}(\rho, \theta)\right] \\
& +\sum_{l-p \leqslant-M-1}\left[\mathcal{L}_{p}^{0}\left(u_{i+p-l}^{k}\right) y_{p, l}(\rho, \theta)\right]
\end{aligned}\right.
$$

the term in the second line (once again convergence details has to be checked) is bounded by $C \rho^{-M-1}$. Next we choose $M=n-i$ so that we can write

$$
\left\{\begin{aligned}
\mathcal{U}_{i}^{k}\left(\frac{r}{\varepsilon}, \theta\right)= & \sum_{l-p \geqslant-n+i}\left[\mathcal{L}_{p}^{0}\left(u_{i+p-l}^{k}\right) y_{p, l}\left(\frac{r}{\varepsilon}, \theta\right)\right]+\sum_{p, l}\left[\left(\mathcal{L}_{p}^{1}\left(u_{i-p-l}^{k}\right)+\frac{2}{\pi} \mathcal{L}_{p}^{0}\left(u_{i-p-l}^{k-1}\right)\right) j_{p, l}\left(\frac{r}{\varepsilon}, \theta\right)\right] \\
& +\delta \mathcal{U}_{i}^{k}\left(\frac{r}{\varepsilon}, \theta\right)
\end{aligned}\right.
$$

where

$$
\left|\delta \mathcal{U}_{i}^{k}\left(\frac{r}{\varepsilon}, \theta\right)\right| \leqslant C\left(\frac{\varepsilon}{\eta_{H}}\right)^{n-i+1} \quad \text { for } r \geqslant \eta_{H}(\varepsilon) .
$$

Next, we substitute (3.86) and (3.91) in (3.79) to obtain ${ }^{2}$

$$
\mathcal{E}_{n}^{\varepsilon}(r, \theta)=\mathrm{S}_{n}^{\varepsilon}(r, \theta)+\mathrm{R}_{n}^{\varepsilon}(r, \theta)
$$

with

$$
\mathrm{R}_{n}^{\varepsilon}(r, \theta)=\sum_{i=0}^{n} \sum_{k=0}^{i}\left(\frac{\omega \varepsilon}{2}\right)^{i} \log ^{k}\left(\frac{\omega \varepsilon}{2}\right)\left(\delta \mathcal{U}_{i}^{k}\left(\frac{r}{\varepsilon}, \theta\right)-\delta u_{i}^{k}(r, \theta)\right)
$$

\footnotetext{
${ }^{2} \mathrm{~A}$ tedious study of the series $\mathrm{A}_{n}^{\varepsilon}, \mathrm{B}_{n}^{\varepsilon}, \mathrm{C}_{n}^{\varepsilon}$, and $\mathrm{D}_{n}^{\varepsilon}$ shows that $\mathrm{S}_{n}^{\varepsilon}$ only contains a finite number of nonvanishing terms. Hence, the following algebraic transforms are allowed without convergence consideration.
} 


$$
\left\{\begin{array}{l}
\mathrm{S}_{n}^{\varepsilon}(r, \theta)=\mathrm{A}_{n}^{\varepsilon}(r, \theta)+\mathrm{B}_{n}^{\varepsilon}(r, \theta)-\mathrm{C}_{n}^{\varepsilon}(r, \theta)-\mathrm{D}_{n}^{\varepsilon}(r, \theta), \quad \text { with } \\
\mathrm{A}_{n}^{\varepsilon}(r, \theta)=\sum_{i=0}^{n} \sum_{k=0}^{i}\left\{\left(\frac{\omega \varepsilon}{2}\right)^{i}\left(\log \frac{\omega \varepsilon}{2}\right)^{k} \sum_{l-p \geqslant i-n}\left[\mathcal{L}_{p}^{0}\left(u_{i+p-l}^{k}\right) y_{p, l}\left(\frac{r}{\varepsilon}, \theta\right)\right]\right\} \\
\mathrm{B}_{n}^{\varepsilon}(r, \theta)=\sum_{i=0}^{n} \sum_{k=0}^{i}\left\{\left(\frac{\omega \varepsilon}{2}\right)^{i}\left(\log \frac{\omega \varepsilon}{2}\right)^{k} \sum_{p, l}\left[\left(\mathcal{L}_{p}^{1}\left(u_{i-p-l}^{k}\right)+\frac{2}{\pi} \mathcal{L}_{p}^{0}\left(u_{i-p-l}^{k-1}\right)\right) j_{p, l}\left(\frac{r}{\varepsilon}, \theta\right)\right]\right\} \\
\mathrm{C}_{n}^{\varepsilon}(r, \theta)=\sum_{i=0}^{n} \sum_{k=0}^{i}\left\{\left(\frac{\omega \varepsilon}{2}\right)^{i}\left(\log \frac{\omega \varepsilon}{2}\right)^{k} \sum_{l-p \leqslant n-i}\left[\mathcal{L}_{p}^{0}\left(u_{i}^{k}\right) y_{p, l}\left(\frac{\omega r}{2}, \theta\right)\right]\right\} \\
\mathrm{D}_{n}^{\varepsilon}(r, \theta)=\sum_{i=0}^{n} \sum_{k=0}^{i}\left\{\left(\frac{\omega \varepsilon}{2}\right)^{i}\left(\log \frac{\omega \varepsilon}{2}\right)^{k} \sum_{p+l \leqslant n-i}\left[\mathcal{L}_{p}^{1}\left(u_{i}^{k}\right) j_{p, l}\left(\frac{\omega r}{2}, \theta\right)\right]\right\}
\end{array}\right.
$$

It follows from (3.87) and (3.92) that for $\eta_{H} \leqslant r \leqslant 2 \eta_{H}$

$$
\left|\mathrm{R}_{n}^{\varepsilon}(r, \theta)\right| \leqslant C \sum_{i=0}^{n} \sum_{k=0}^{i}\left(\frac{\omega \varepsilon}{2}\right)^{i}\left|\log \frac{\omega \varepsilon}{2}\right|^{k}\left[\left(\frac{\omega \eta_{H}}{2}\right)^{n-i+1}\left|\log \frac{\omega \eta_{H}}{2}\right|+\left(\frac{\varepsilon}{\eta_{H}}\right)^{n-i+1}\right]
$$

Since $1 \leqslant \log \frac{\omega \eta_{H}}{2}$ and $\frac{\omega \varepsilon}{2} \leqslant \frac{\omega \eta_{H}}{2}$, we have

$$
\left\{\begin{aligned}
\left|\mathrm{R}_{n}^{\varepsilon}(r, \theta)\right| & \leqslant C \sum_{i=0}^{n} \sum_{k=0}^{i}\left(\frac{\omega \eta_{H}}{2}\right)^{i}\left|\log \frac{\omega \varepsilon}{2}\right|^{k}\left|\log \frac{\omega \eta_{H}}{2}\right|\left[\left(\frac{\omega \eta_{H}}{2}\right)^{n-i+1}+\left(\frac{\varepsilon}{\eta_{H}}\right)^{n-i+1}\right] \\
& \leqslant C \sum_{i=0}^{n} \sum_{k=0}^{i}\left|\log \frac{\omega \varepsilon}{2}\right|^{k}\left|\log \frac{\omega \eta_{H}}{2}\right|\left[\left(\frac{\omega \eta_{H}}{2}\right)^{n+1}+\left(\frac{\omega \eta_{H}}{2}\right)^{i}\left(\frac{\varepsilon}{\eta_{H}}\right)^{n-i+1}\right] \\
& \leqslant C \sum_{i=0}^{n} \sum_{k=0}^{i}\left|\log \frac{\omega \varepsilon}{2}\right|^{k}\left|\log \frac{\omega \eta_{H}}{2}\right|\left[\left(\frac{\omega \eta_{H}}{2}\right)^{n+1}+\left(\frac{\varepsilon}{\eta_{H}}\right)^{n+1}\right]
\end{aligned}\right.
$$

where we have used $\left(\frac{\omega \eta_{H}}{2}\right)^{i}\left(\frac{\varepsilon}{\eta_{H}}\right)^{n-i+1} \leqslant\left(\frac{\omega \eta_{H}}{2}\right)^{n+1}+\left(\frac{\varepsilon}{\eta_{H}}\right)^{n+1}$.

Finally, with $\left|\log \frac{\omega \varepsilon}{2}\right|^{k} \leqslant\left|\log \frac{\omega \varepsilon}{2}\right|^{n}$, we get

$$
\left|\mathrm{R}_{n}^{\varepsilon}(r, \theta)\right| \leqslant C\left|\log \frac{\omega \eta_{H}}{2}\right|\left|\log \frac{\omega \varepsilon}{2}\right|^{n}\left[\left(\frac{\omega \eta_{H}}{2}\right)^{n+1}+\left(\frac{\varepsilon}{\eta_{H}}\right)^{n+1}\right] .
$$

To conclude the proof, it suffices to check that $S_{n}^{\varepsilon}$ vanishes identically. This is due to the fact that $S_{n}^{\varepsilon}$ is composed of terms that cancel each others thanks to the matching conditions. The verification of this is essentially a matter of transforming and manipulating (3.95) defining $\mathrm{A}_{n}^{\varepsilon}, \mathrm{B}_{n}^{\varepsilon}, \mathrm{C}_{n}^{\varepsilon}$, and $\mathrm{D}_{n}^{\varepsilon}$. There is not a unique way to do it but we present below the one which appear to us as the simplest.

The first observation is that in (3.95), $\sum_{k=0}^{i}$ can be replaced by $\sum_{k=-\infty}^{+\infty}$.

For the sums $\mathbf{C}_{n}^{\varepsilon}$ and $\mathrm{D}_{n}^{\varepsilon}$ this is simply a consequence of the convention $(2.17), u_{i}^{k} \equiv 0$ for all $(i, k) \notin \mathbf{J}$.

For $\mathrm{A}_{n}^{\varepsilon}$ this follows from the property $(2.30)$, namely $\mathcal{L}_{p}^{0}\left(u_{i}^{k}\right)=0$ for $i-k \leqslant p$. Indeed,

$$
\begin{cases}\text { if } k<0, & \mathcal{L}_{p}^{0}\left(u_{i+p-l}^{k}\right)=0 \text { by }(2.17) \\ \text { if } k>i, & \mathcal{L}_{p}^{0}\left(u_{i+p-l}^{k}\right)=0 \text { since }(i+p-l)-k=p-l+(i-k) \leqslant p .\end{cases}
$$


Finally for the sum $\mathrm{B}_{n}^{\varepsilon}$, the same argument works. The details are left to the reader. Therefore we can write

$$
\left\{\begin{aligned}
\mathrm{A}_{n}^{\varepsilon}(r, \theta) & =\sum_{k=-\infty}^{+\infty} \sum_{i=0}^{n} \sum_{l-p \geqslant i-n}\left\{\left(\frac{\omega \varepsilon}{2}\right)^{i}\left(\log \frac{\omega \varepsilon}{2}\right)^{k} \mathcal{L}_{p}^{0}\left(u_{i+p-l}^{k}\right) y_{p, l}\left(\frac{r}{\varepsilon}, \theta\right)\right\} \\
\mathrm{B}_{n}^{\varepsilon}(r, \theta) & =\sum_{k=-\infty}^{+\infty} \sum_{i=0}^{n} \sum_{l, p}\left\{\left(\frac{\omega \varepsilon}{2}\right)^{i}\left(\log \frac{\omega \varepsilon}{2}\right)^{k}\left(\mathcal{L}_{p}^{1}\left(u_{i-p-l}^{k}\right)+\frac{2}{\pi} \mathcal{L}_{p}^{0}\left(u_{i-p-l}^{k-1}\right)\right) j_{p, l}\left(\frac{r}{\varepsilon}, \theta\right)\right\} \\
\mathrm{C}_{n}^{\varepsilon}(r, \theta) & =\sum_{k=-\infty}^{+\infty} \sum_{i=0}^{n} \sum_{l-p \leqslant n-i}\left\{\left(\frac{\omega \varepsilon}{2}\right)^{i}\left(\log \frac{\omega \varepsilon}{2}\right)^{k} \mathcal{L}_{p}^{0}\left(u_{i}^{k}\right) y_{p, l}\left(\frac{\omega r}{2}, \theta\right)\right\} \\
\mathrm{D}_{n}^{\varepsilon}(r, \theta) & =\sum_{k=-\infty}^{+\infty} \sum_{i=0}^{n} \sum_{l+p \leqslant n-i}\left\{\left(\frac{\omega \varepsilon}{2}\right)^{i}\left(\log \frac{\omega \varepsilon}{2}\right)^{k} \mathcal{L}_{p}^{1}\left(u_{i}^{k}\right) j_{p, l}\left(\frac{\omega r}{2}, \theta\right)\right\}
\end{aligned}\right.
$$

To conclude, we simply has to transform the expression $\mathrm{A}_{n}^{\varepsilon}$ and $\mathrm{D}_{n}^{\varepsilon}$ by using a change of index of summation and the properties of $j_{p, l}$ and $y_{p, l}$.

Transformation of $\mathrm{D}_{n}^{\varepsilon}$. We observe that

$$
\left\{(i, p, l) \in \mathbb{N}^{3} \mid i \leqslant n, p+l \leqslant n-i\right\}=\left\{(i, p, l) \in \mathbb{N}^{3} \mid i+p+l \leqslant n\right\}
$$

so that

$$
\mathrm{D}_{n}^{\varepsilon}(r, \theta)=\sum_{k=-\infty}^{+\infty} \sum_{l+p \leqslant n} \sum_{i=0}^{n-l-p}\left\{\left(\frac{\omega \varepsilon}{2}\right)^{i}\left(\log \frac{\omega \varepsilon}{2}\right)^{k} \mathcal{L}_{p}^{1}\left(u_{i}^{k}\right) j_{p, l}\left(\frac{\omega r}{2}, \theta\right)\right\} .
$$

The function $j_{p, l}$, given by $(2.5)$, is homogeneous of degree $p+l$

$$
\begin{gathered}
j_{p, l}(\omega r / 2, \theta)=(\omega \varepsilon / 2)^{p+l} j_{p, l}(r / \varepsilon, \theta) . \\
\mathrm{D}_{n}^{\varepsilon}(r, \theta)=\sum_{k=-\infty}^{+\infty} \sum_{l+p \leqslant n} \sum_{i=0}^{n-l-p}\left\{\left(\frac{\omega \varepsilon}{2}\right)^{i+p+l}\left(\log \frac{\omega \varepsilon}{2}\right)^{k} \mathcal{L}_{p}^{1}\left(u_{i}^{k}\right) j_{p, l}\left(\frac{r}{\varepsilon}, \theta\right)\right\} .
\end{gathered}
$$

Using the change of index $i+p+l \rightarrow i$, we get

$$
\mathrm{D}_{n}^{\varepsilon}(r, \theta)=\sum_{k=-\infty}^{+\infty} \sum_{l+p \leqslant n} \sum_{i=l+p}^{n}\left\{\left(\frac{\omega \varepsilon}{2}\right)^{i}\left(\log \frac{\omega \varepsilon}{2}\right)^{k} \mathcal{L}_{p}^{1}\left(u_{i-p-l}^{k}\right) j_{p, l}\left(\frac{r}{\varepsilon}, \theta\right)\right\} .
$$

Since

$$
\left\{(i, p, l) \in \mathbb{N}^{3} \mid p+l \leqslant n, l+p \leqslant i \leqslant n\right\}=\left\{(i, p, l) \in \mathbb{N}^{3} \mid i \leqslant n, l+p \leqslant i\right\},
$$

we have

$$
\mathrm{D}_{n}^{\varepsilon}(r, \theta)=\sum_{k=-\infty}^{+\infty} \sum_{i=0}^{n} \sum_{l+p \leqslant i}\left\{\left(\frac{\omega \varepsilon}{2}\right)^{i}\left(\log \frac{\omega \varepsilon}{2}\right)^{k} \mathcal{L}_{p}^{1}\left(u_{i-p-l}^{k}\right) j_{p, l}\left(\frac{r}{\varepsilon}, \theta\right)\right\}
$$

which we can rewrite, since $u_{i-p-l}^{k} \equiv 0$ for $p+l>i$, as

$$
\mathrm{D}_{n}^{\varepsilon}(r, \theta)=\sum_{k=-\infty}^{+\infty} \sum_{i=0}^{n} \sum_{l, p}\left\{\left(\frac{\omega \varepsilon}{2}\right)^{i}\left(\log \frac{\omega \varepsilon}{2}\right)^{k} \mathcal{L}_{p}^{1}\left(u_{i-p-l}^{k}\right) j_{p, l}\left(\frac{r}{\varepsilon}, \theta\right)\right\} .
$$


Computation of $\mathrm{A}_{n}^{\varepsilon}$. Since $y_{p, l}$ is given by (2.5), we obtain

$$
y_{p, l}\left(\frac{r}{\varepsilon}, \theta\right)=\left(\frac{\omega \varepsilon}{2}\right)^{p-l} y_{p, l}\left(\frac{\omega r}{2}, \theta\right)-\frac{2}{\pi} \log \left(\frac{\omega \varepsilon}{2}\right) j_{p,-2 p+l}\left(\frac{r}{\varepsilon}, \theta\right) .
$$

Hence, $\mathrm{A}_{n}^{\varepsilon}$ can be transformed to

$$
\left\{\begin{aligned}
\mathrm{A}_{n}^{\varepsilon}(r, \theta)= & \sum_{k=-\infty}^{+\infty} \sum_{i=0}^{n} \sum_{p-l \leqslant n-i}\left[\left(\frac{\omega \varepsilon}{2}\right)^{i+p-l}\left(\log \frac{\omega \varepsilon}{2}\right)^{k} \mathcal{L}_{p}^{0}\left(u_{i+p-l}^{k}\right) y_{p, l}\left(\frac{\omega r}{2}, \theta\right)\right] \\
& -\frac{2}{\pi} \sum_{i=0}^{n} \sum_{k=-\infty}^{+\infty} \sum_{p-l \leqslant n-i}\left[\left(\frac{\omega \varepsilon}{2}\right)^{i}\left(\log \frac{\omega \varepsilon}{2}\right)^{k+1} \mathcal{L}_{p}^{0}\left(u_{i+p-l}^{k}\right) j_{p,-2 p+l}\left(\frac{r}{\varepsilon}, \theta\right)\right]
\end{aligned}\right.
$$

Rewriting the second sum, we get

$$
\left\{\begin{aligned}
\mathrm{A}_{n}^{\varepsilon}(r, \theta)= & \sum_{i=0}^{n} \sum_{k=-\infty}^{+\infty} \sum_{p-l \leqslant n-i}\left[\left(\frac{\omega \varepsilon}{2}\right)^{i+p-l}\left(\log \frac{\omega \varepsilon}{2}\right)^{k} \mathcal{L}_{p}^{0}\left(u_{i+p-l}^{k}\right) y_{p, l}\left(\frac{\omega r}{2}, \theta\right)\right] \\
& -\frac{2}{\pi} \sum_{i=0}^{n} \sum_{k=-\infty}^{+\infty} \sum_{p=0}^{+\infty}\left\{\sum_{l \geqslant \max (0, p-(n-i))}\left[\left(\frac{\omega \varepsilon}{2}\right)^{i}\left(\log \frac{\omega \varepsilon}{2}\right)^{k+1} \mathcal{L}_{p}^{0}\left(u_{i+p-l}^{k}\right) j_{p,-2 p+l}\left(\frac{r}{\varepsilon}, \theta\right)\right]\right\} .
\end{aligned}\right.
$$

Since for all $l$ such that $\max (0, p-(n-i)) \leqslant l<2 p, j_{p, l-2 p} \equiv 0$, one can restrict the last sum of the second line to the $l \geqslant 2 p$

$$
\left\{\begin{aligned}
\mathrm{A}_{n}^{\varepsilon}(r, \theta)= & \sum_{k=-\infty}^{+\infty} \sum_{i=0}^{n} \sum_{p-l \leqslant n-i}\left[\left(\frac{\omega \varepsilon}{2}\right)^{i+p-l}\left(\log \frac{\omega \varepsilon}{2}\right)^{k} \mathcal{L}_{p}^{0}\left(u_{i+p-l}^{k}\right) y_{p, l}\left(\frac{\omega r}{2}, \theta\right)\right] \\
& -\frac{2}{\pi} \sum_{k=-\infty}^{+\infty} \sum_{i=0}^{n} \sum_{p=0}^{+\infty} \sum_{l \geqslant 2 p}\left[\left(\frac{\omega \varepsilon}{2}\right)^{i}\left(\log \frac{\omega \varepsilon}{2}\right)^{k+1} \mathcal{L}_{p}^{0}\left(u_{i+p-l}^{k}\right) j_{p,-2 p+l}\left(\frac{r}{\varepsilon}, \theta\right)\right]
\end{aligned}\right.
$$

Applying the change of index of summation

- $i+p-l \rightarrow i$ in the first sum

- $k+1 \rightarrow k,-2 p+l \rightarrow l$ in the second sum

$\mathrm{A}_{n}^{\varepsilon}$ can be expressed as

$$
\left\{\begin{aligned}
\mathrm{A}_{n}^{\varepsilon}(r, \theta)= & \sum_{k=-\infty}^{+\infty} \sum_{i=0}^{n} \sum_{l-p \leqslant n-i}\left[\left(\frac{\omega \varepsilon}{2}\right)^{i}\left(\log \frac{\omega \varepsilon}{2}\right)^{k} \mathcal{L}_{p}^{0}\left(u_{i}^{k}\right) y_{p, l}\left(\frac{\omega r}{2}, \theta\right)\right] \\
& -\frac{2}{\pi} \sum_{k=-\infty}^{+\infty} \sum_{i=0}^{n} \sum_{p, l}\left[\left(\frac{\omega \varepsilon}{2}\right)^{i}\left(\log \frac{\omega \varepsilon}{2}\right)^{k} \mathcal{L}_{p}^{0}\left(u_{i-p-l}^{k-1}\right) j_{p, l}\left(\frac{r}{\varepsilon}, \theta\right)\right]
\end{aligned}\right.
$$

Conclusion. Looking at the expression of $\mathrm{B}_{n}^{\varepsilon}(r, \theta)$ and $\mathbf{C}_{n}^{\varepsilon}(r, \theta)$ in (3.100), of $\mathbf{A}_{n}^{\varepsilon}(r, \theta)$ in (3.113), and of $\mathrm{D}_{n}^{\varepsilon}(r, \theta)$ in (3.108), the reader can check that

$$
\mathrm{A}_{n}^{\varepsilon}(r, \theta)+\mathrm{D}_{n}^{\varepsilon}(r, \theta)=\mathrm{B}_{n}^{\varepsilon}(r, \theta)+\mathrm{C}_{n}^{\varepsilon}(r, \theta)
$$

and the proof is complete. 


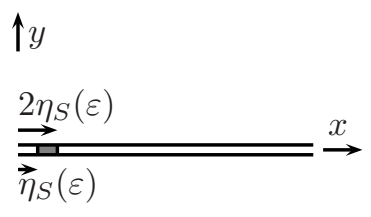

$\uparrow Y$

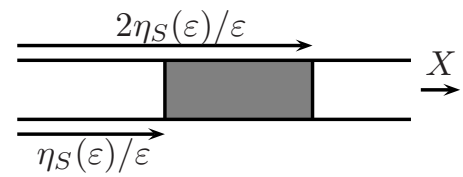

FigurE 9. The intermediate zone between slot-field and near-field.

\subsubsection{Matching error between the slot-field and the near-field}

We denote by $E_{n}^{\varepsilon}$ the matching error between the slot-field and the near-field, i.e. the function defined in the domain, see Figure 9,

$$
C_{S}^{\varepsilon}=\left\{(x, y) \in \Omega_{S}^{\varepsilon} \mid \eta_{S}(\varepsilon) \leqslant x \leqslant 2 \eta_{S}(\varepsilon)\right\}
$$

by

$$
E_{n}^{\varepsilon}(x, y)=\sum_{i=0}^{n} \sum_{k=0}^{i}\left(\frac{\omega \varepsilon}{2}\right)^{i}\left(\log \frac{\omega \varepsilon}{2}\right)^{k} \mathcal{U}_{i}^{k}(x / \varepsilon, y / \varepsilon)-\sum_{i=0}^{n} \sum_{k=0}^{i}\left(\frac{\omega \varepsilon}{2}\right)^{i}\left(\log \frac{\omega \varepsilon}{2}\right)^{k} U_{i}^{k}(x) .
$$

As it will be shown in Lemma 3.7, this quantity is small in the intermediate region $C_{S}^{\varepsilon}$ which tends to zero in the physical coordinates and to infinity in the near-field coordinates (see (3.12)).

Lemma 3.7. For any $n$ and $m$ in $\mathbb{N}$, there exist a constant $C_{n, m}=C_{n, m}(f, \omega) \in \mathbb{R}$ and $\varepsilon_{0}>0$ satisfying, for all $0<\varepsilon<\varepsilon_{0}$ :

$$
\left\{\begin{array}{l}
\left\|E_{n}^{\varepsilon}\right\|_{L^{\infty}\left(C_{S}^{\varepsilon}\right)} \leqslant C_{n, m}\left(\log \frac{\omega \varepsilon}{2}\right)^{n}\left[\left(\frac{\omega \eta_{S}}{2}\right)^{n+1}+\left(\frac{\varepsilon}{\eta_{S}}\right)^{m}\right], \\
\left\|\nabla E_{n}^{\varepsilon}\right\|_{L^{\infty}\left(C_{S}^{\varepsilon}\right)} \leqslant C_{n, m}\left(\log \frac{\omega \varepsilon}{2}\right)^{n} \frac{1}{\eta_{S}}\left[\left(\frac{\omega \eta_{S}}{2}\right)^{n+1}+\left(\frac{\varepsilon}{\eta_{S}}\right)^{m}\right] .
\end{array}\right.
$$

Proof. The proof of this lemma is rather similar to the proof of Lemma 3.6. We only prove the first line of $(3.117)$, the second one is left to the reader.

Let $(i, k) \in \mathbf{J}$ with $i \leqslant n$.

Due to (2.21) and to Taylor expansion of $\exp (\mathbf{i} \omega x)$, one has

$$
U_{i}^{k}(x)=U_{i}^{k}(0) \exp \mathbf{i} \omega x=\sum_{l=0}^{+\infty} U_{i}^{k}(0) \frac{(\mathbf{i} \omega x)^{l}}{l !}
$$

Truncating this series at order $n-i$, one gets

$$
U_{i}^{k}(x)=\sum_{l=0}^{n-i} U_{i}^{k}(0) \frac{(\mathbf{i} \omega x)^{l}}{l !}+\delta U_{i}^{k}(x), \quad \text { with } \quad\left|\delta U_{i}^{k}(x)\right| \leqslant C_{n}\left(\frac{\omega x}{2}\right)^{n-i+1} .
$$

The near-field $\mathcal{U}_{i}^{k}$ is expanded using (2.28)

$$
\left\{\begin{array}{l}
\mathcal{U}_{i}^{k}(X, Y)=\sum_{l=0}^{i-k} U_{i-l}^{k}(0) \frac{(2 \mathbf{i} X)^{l}}{l !}+\delta \mathcal{U}_{i}^{k}(X, Y) \\
\text { with }\left|\delta \mathcal{U}_{i}^{k}(X, Y)\right| \leqslant C_{m} X^{-m}, \quad \forall m \in \mathbb{N}
\end{array}\right.
$$


Given

the matching error $E_{n}^{\varepsilon}$ can be decomposed in

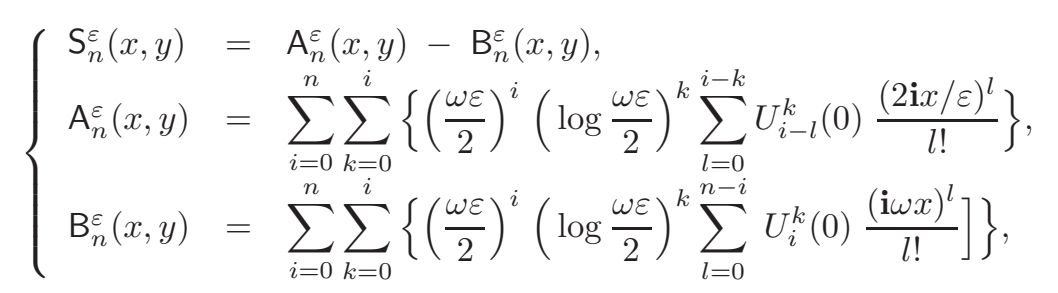

$$
E_{n}^{\varepsilon}(x, y)=\mathrm{S}_{n}^{\varepsilon}(x, y)+\sum_{i=0}^{n} \sum_{k=0}^{i}\left(\frac{\omega \varepsilon}{2}\right)^{i}\left(\log \frac{\omega \varepsilon}{2}\right)^{k}\left(\delta \mathcal{U}_{i}^{k}\left(\frac{x}{\varepsilon}, \frac{y}{\varepsilon}\right)-\delta U_{i}^{k}(x)\right), \quad(x, y) \in C_{S}^{\varepsilon} .
$$

In the intermediate zone $C_{S}^{\varepsilon}$, the remainders are bounded as follows

$$
\left\{\begin{array}{l}
\left|\delta \mathcal{U}_{i, k}\left(\frac{x}{\varepsilon}, \frac{y}{\varepsilon}\right)\right| \leqslant C_{m}\left(\frac{\varepsilon}{\eta_{S}}\right)^{m}, \quad \forall m \in \mathbb{N} \\
\left|\delta U_{i, k}(x)\right| \leqslant C_{n}\left(\frac{\omega \eta_{S}}{2}\right)^{n-i+1}
\end{array}\right.
$$

Hence, one has similarly to $(3.98)$

$$
\left\{\begin{aligned}
\left|E_{n}^{\varepsilon}(x, y)\right| & \leqslant\left|\mathrm{S}_{n}^{\varepsilon}(x, y)\right|+C_{m, n} \sum_{i=0}^{n} \sum_{k=0}^{i}\left(\frac{\omega \varepsilon}{2}\right)^{i}\left|\log \frac{\omega \varepsilon}{2}\right|^{k}\left[\left(\frac{\omega \eta_{S}}{2}\right)^{n-i+1}+\left(\frac{\varepsilon}{\eta_{S}}\right)^{m}\right] \\
& \leqslant\left|\mathrm{S}_{n}^{\varepsilon}(x, y)\right|+C_{m, n}\left|\log \frac{\omega \varepsilon}{2}\right|^{n}\left[\left(\frac{\omega \eta_{S}}{2}\right)^{n+1}+\left(\frac{\varepsilon}{\eta_{S}}\right)^{m}\right] .
\end{aligned}\right.
$$

To conclude the proof, it suffices to show that $\mathrm{S}_{n}^{\varepsilon}$ is equal to zero. We show that $\mathrm{A}_{n}^{\varepsilon}=\mathrm{B}_{n}^{\varepsilon}$, see (3.121). Starting from $\mathrm{A}_{n}^{\varepsilon}$, we get with two discreet Fubiny formulas for $k$ and $l$ (second line) and $i$ and $l$ (third line)

$$
\left\{\begin{aligned}
\mathrm{A}_{n}^{\varepsilon}(x, y) & =\sum_{i=0}^{n} \sum_{k=0}^{i} \sum_{l=0}^{i-k}\left[\left(\frac{\omega \varepsilon}{2}\right)^{i}\left(\log \frac{\omega \varepsilon}{2}\right)^{k} U_{i-l}^{k}(0) \frac{(2 \mathbf{i} x / \varepsilon)^{l}}{l !}\right] \\
& =\sum_{i=0}^{n} \sum_{l=0}^{i} \sum_{k=0}^{i-l}\left[\left(\frac{\omega \varepsilon}{2}\right)^{i-l}\left(\log \frac{\omega \varepsilon}{2}\right)^{k} U_{i-l}^{k}(0) \frac{(\mathbf{i} \omega x)^{l}}{l !}\right] \\
& =\sum_{l=0}^{n} \sum_{i=l}^{n} \sum_{k=0}^{i-l}\left[\left(\frac{\omega \varepsilon}{2}\right)^{i-l}\left(\log \frac{\omega \varepsilon}{2}\right)^{k} U_{i-l}^{k}(0) \frac{(\mathbf{i} \omega x)^{l}}{l !}\right] .
\end{aligned}\right.
$$

The change of index of summation $i-l \rightarrow i$ leads to

$$
\left\{\begin{aligned}
\mathrm{A}_{n}^{\varepsilon}(x, y) & =\sum_{l=0}^{n} \sum_{i=0}^{n-l} \sum_{k=0}^{i}\left[\left(\frac{\omega \varepsilon}{2}\right)^{i}\left(\log \frac{\omega \varepsilon}{2}\right)^{k} U_{i}^{k}(0) \frac{(\mathbf{i} \omega x)^{l}}{l !}\right] \\
& =\sum_{i=0}^{n} \sum_{l=0}^{n-i} \sum_{k=0}^{i}\left[\left(\frac{\omega \varepsilon}{2}\right)^{i}\left(\log \frac{\omega \varepsilon}{2}\right)^{k} U_{i}^{k}(0) \frac{(\mathbf{i} \omega x)^{l}}{l !}\right] \\
& =\sum_{i=0}^{n} \sum_{k=0}^{i} \sum_{l=0}^{n-i}\left[\left(\frac{\omega \varepsilon}{2}\right)^{i}\left(\log \frac{\omega \varepsilon}{2}\right)^{k} U_{i}^{k}(0) \frac{(\mathbf{i} \omega x)^{l}}{l !}\right]
\end{aligned}\right.
$$

where we have used again two discreet Fubiny formulas. Comparing with (3.121), it follows

$$
\mathrm{A}_{n}^{\varepsilon}(x, y)=\mathrm{B}_{n}^{\varepsilon}(x, y) .
$$


This ends the proof.

\subsubsection{Proof of Lemma 3.5}

We will obtain the consistency estimate (3.45) by duality

$$
\left\|\mathbf{A}_{b}^{\varepsilon} \widetilde{u}_{n}^{\varepsilon}-L_{b}^{\varepsilon}\right\|_{H^{1}\left(\Omega_{\varepsilon}^{b}\right)} \leqslant \sup _{v \in H^{1}\left(\Omega_{\varepsilon}^{b}\right) \backslash\{0\}} \frac{\left(\mathbf{A}_{b}^{\varepsilon} \widetilde{u}_{n}^{\varepsilon}-L_{b}^{\varepsilon} ; v\right)_{H^{1}\left(\Omega_{\varepsilon}^{b}\right)}}{\|v\|_{H^{1}\left(\Omega_{\varepsilon}^{b}\right)}} .
$$

By definition of $\mathbf{A}_{b}^{\varepsilon}$ and $L_{b}^{\varepsilon}$ — see (3.39), (3.41), and (3.42) - one has

$$
\left\{\begin{aligned}
\left(\mathbf{A}_{b}^{\varepsilon} \widetilde{u}_{n}^{\varepsilon}-L_{b}^{\varepsilon} ; v\right)_{H^{1}\left(\Omega_{\varepsilon}^{b}\right)}= & \int_{\Omega_{b}^{\varepsilon}}\left(\nabla \widetilde{u}_{n}^{\varepsilon} \nabla v-\omega^{2} \widetilde{u}_{n}^{\varepsilon} \bar{v}-f \bar{v}\right) \\
& +\left\langle T_{\varepsilon, \delta} \widetilde{u}_{n}^{\varepsilon} ; v\right\rangle_{\Sigma^{\varepsilon, \delta}}+\left\langle T_{R} \widetilde{u}_{n}^{\varepsilon} ; v\right\rangle_{\Gamma_{R}} .
\end{aligned}\right.
$$

To go further, we shall use some properties of the function $u_{n}^{\varepsilon}$.

Lemma 3.8. The function $\widetilde{u}_{n}^{\varepsilon}$ satisfies

(i) $\quad \Delta \widetilde{u}_{n}^{\varepsilon}+\omega^{2} \widetilde{u}_{n}^{\varepsilon}=-f, \quad$ in $\Omega_{\varepsilon} \backslash B_{N}^{\varepsilon}, \quad$ (see (3.4) for $\left.B_{N}^{\varepsilon}\right)$;

(ii) $\frac{\partial \widetilde{u}_{n}^{\varepsilon}}{\partial n}=0$, on $\partial \Omega_{\varepsilon}$;

(iii) $\quad \frac{\partial \widetilde{u}_{n}^{\varepsilon}}{\partial n}+T_{A} \widetilde{u}_{n}^{\varepsilon}=0, \quad$ on $\Gamma_{A}$;

(iv) $\frac{\partial \widetilde{u}_{n}^{\varepsilon}}{\partial n}+T_{\varepsilon, \delta} \widetilde{u}_{n}^{\varepsilon}=0$, on $\Sigma_{\varepsilon, \delta}$.

Proof. We first notice that the function $u_{H, n}^{\varepsilon}$ defined in $\Omega_{H}$ by (3.1) satisfies

$$
\begin{cases}\Delta u_{H, n}^{\varepsilon}+\omega^{2} u_{H, n}^{\varepsilon}=-f & \text { in } \Omega_{H} \\ \frac{\partial u_{H, n}^{\varepsilon}}{\partial n}+T_{A} u_{H, n}^{\varepsilon}=0 & \text { on } \Gamma_{A} .\end{cases}
$$

This is simply a consequence of the equations satisfied by the $u_{i}^{k}$ 's.

On the other hand, the function $u_{S, n}^{\varepsilon}$ defined in $\Omega_{S}^{\varepsilon}$ by (3.3) satisfies:

$$
\begin{cases}\Delta u_{S, n}^{\varepsilon}+\omega^{2} u_{S, n}^{\varepsilon}=-f \equiv 0, & \text { in } \Omega_{S}^{\varepsilon} \\ \frac{\partial u_{S, n}^{\varepsilon}}{\partial n}+T^{\varepsilon, \delta} u_{S, n}^{\varepsilon}=0, & \text { on } \Sigma_{\varepsilon, \delta}\end{cases}
$$

as a consequence of the properties of the $U_{i}^{k}$ 's.

Thus, (i), (iii), and (iv) are a consequence of (cf. (3.10), Sect. 3.1)

$$
\widetilde{u}_{n}^{\varepsilon}=u_{H, n}^{\varepsilon}, \text { in } \Omega_{H} \backslash B_{H}^{\varepsilon}, \quad \widetilde{u}_{n}^{\varepsilon}=u_{S, n}^{\varepsilon}, \text { in } \Omega_{S}^{\varepsilon} \backslash B_{S}^{\varepsilon} .
$$

Then, to check whether (ii) is also satisfied, it suffices to remark that the Neumann boundary conditions is satisfied by the $u_{i}^{k}$ 's, $U_{i}^{k}$ 's, and $\mathcal{U}_{i}^{k}$ 's

$$
\frac{\partial u_{H, n}^{\varepsilon}}{\partial n}=0 \quad \text { on } \partial \Omega_{\varepsilon} \cap \partial \Omega_{H}, \quad \frac{\partial u_{S, n}^{\varepsilon}}{\partial n}=0 \quad \text { on } \partial \Omega_{\varepsilon} \cap \partial \Omega_{S}^{\varepsilon}, \quad \frac{\partial u_{N, n}^{\varepsilon}}{\partial n}=0 \quad \text { on } \partial \Omega_{N}^{\varepsilon} .
$$

Finally, we use the definition (3.9) of $\widetilde{u}_{n}^{\varepsilon}$, the properties (3.8) of the cut-off functions $\chi_{H}^{\varepsilon}$, and $\chi_{S}^{\varepsilon}$. 
Using Green's formula and Lemma 3.8, we obtain, since $\operatorname{supp}(f) \cap B_{N}^{\varepsilon}=\emptyset$,

$$
\left(\mathbf{A}_{b}^{\varepsilon} \widetilde{u}_{n}^{\varepsilon}-L_{b}^{\varepsilon} ; v\right)_{H^{1}\left(\Omega_{\varepsilon}^{b}\right)}=-\int_{B_{N}^{\varepsilon}}\left(\Delta \widetilde{u}_{n}^{\varepsilon}+\omega^{2} \widetilde{u}_{n}^{\varepsilon}\right) \bar{v}
$$

As a consequence, by definition of $B_{N}^{\varepsilon}$, we have

$$
\left\{\begin{aligned}
\left(\mathbf{A}_{b}^{\varepsilon} \widetilde{u}_{n}^{\varepsilon}-L_{b}^{\varepsilon} ; v\right)_{H^{1}\left(\Omega_{\varepsilon}^{b}\right)} \leqslant & \left\|\Delta \widetilde{u}_{n}^{\varepsilon}+\omega^{2} \widetilde{u}_{n}^{\varepsilon}\right\|_{L^{\infty}\left(B_{H}^{\varepsilon}\right)}\|v\|_{L^{1}\left(B_{H}^{\varepsilon}\right)} \\
& +\left\|\Delta \widetilde{u}_{n}^{\varepsilon}+\omega^{2} \widetilde{u}_{n}^{\varepsilon}\right\|_{L^{\infty}\left(B_{S}^{\varepsilon}\right)}\|v\|_{L^{1}\left(B_{S}^{\varepsilon}\right)}
\end{aligned}\right.
$$

Finally, the consistency estimate (3.45) of Lemma 3.5 is a consequence of the following two lemmas.

Lemma 3.9. One has the estimates

$$
\left\{\begin{array}{l}
\text { (i) } \quad\left\|\Delta \widetilde{u}_{n}^{\varepsilon}+\omega^{2} \widetilde{u}_{n}^{\varepsilon}\right\|_{L^{\infty}\left(B_{H}^{\varepsilon}\right)} \leqslant \frac{C}{\eta_{H}^{2}}\left|\log \frac{\omega \varepsilon}{2}\right|^{n}\left|\log \frac{\omega \eta_{H}}{2}\right|\left[\left(\frac{\omega \eta_{H}}{2}\right)^{n+1}+\left(\frac{\varepsilon}{\eta_{H}}\right)^{n+1}\right], \\
\text { (ii) } \quad\left\|\Delta \widetilde{u}_{n}^{\varepsilon}+\omega^{2} \widetilde{u}_{n}^{\varepsilon}\right\|_{L^{\infty}\left(B_{S}^{\varepsilon}\right)} \leqslant \frac{C}{\eta_{S}^{2}}\left|\log \frac{\omega \varepsilon}{2}\right|^{n+1}\left[\left(\frac{\omega \eta_{S}}{2}\right)^{n+1}+\left(\frac{\varepsilon}{\eta_{S}}\right)^{m}\right] .
\end{array}\right.
$$

Lemma 3.10. There exists a constant $C_{R}$ independent of $\varepsilon$ such that

$$
\forall v \in H^{1}\left(B_{R}\right), \quad\|v\|_{L^{1}\left(B_{H}^{\varepsilon}\right)} \leqslant C\left[\eta_{H}(\varepsilon)\right]^{2} \sqrt{\left|\log \eta_{H}(\varepsilon)\right|}\|v\|_{H^{1}\left(B_{R}\right)} .
$$

There exists a constant $C_{\delta}$ independent of $\varepsilon$ such that

$$
\forall v \in H^{1}\left(S_{\delta}^{\varepsilon}\right), \quad\|v\|_{L^{1}\left(B_{S}^{\varepsilon}\right)} \leqslant C \eta_{s}(\varepsilon) \sqrt{\varepsilon}\|v\|_{H^{1}\left(S_{\delta}^{\varepsilon}\right)} .
$$

Proof of Lemma 3.9. We first prove (3.136)(i). In the domain $\Omega_{H}$, we have

$$
\widetilde{u}_{n}^{\varepsilon}=\chi_{H}^{\varepsilon} u_{H, n}^{\varepsilon}+\left(1-\chi_{H}^{\varepsilon}\right) u_{N, n}^{\varepsilon} .
$$

In $B_{H}^{\varepsilon}, \Delta u_{H, n}^{\varepsilon}+\omega^{2} u_{H, n}^{\varepsilon}=0$, and, thus, we obtain

$$
\Delta \widetilde{u}_{n}^{\varepsilon}+\omega^{2} \widetilde{u}_{n}^{\varepsilon}=-\Delta \chi_{H}^{\varepsilon} \mathcal{E}_{n}^{\varepsilon}-2 \nabla \chi_{H}^{\varepsilon} \nabla \mathcal{E}_{n}^{\varepsilon}+\left(1-\chi_{H}^{\varepsilon}\right)\left(\Delta u_{N, n}^{\varepsilon}+\omega^{2} u_{N, n}^{\varepsilon}\right) .
$$

By definition of $\chi_{H}^{\varepsilon}(3.6)$, we have

$$
\left\|\nabla \chi_{\varepsilon}^{H}\right\|_{L^{\infty}\left(B_{H}^{\varepsilon}\right)} \leqslant \frac{C}{\eta_{H}} \quad \text { and } \quad\left\|\Delta \chi_{\varepsilon}^{H}\right\|_{L^{\infty}\left(B_{H}^{\varepsilon}\right)} \leqslant \frac{C}{\eta_{H}^{2}} .
$$

Moreover, the definition (3.2) of $u_{N, n}^{\varepsilon}$ leads to

$$
\left\{\begin{aligned}
\left(\Delta u_{N, n}^{\varepsilon}+\omega^{2} u_{N, n}^{\varepsilon}\right)(\mathbf{x})= & \frac{1}{\varepsilon^{2}} \sum_{i=0}^{n} \sum_{k=0}^{i}\left(\frac{\omega \varepsilon}{2}\right)^{i}\left(\log \frac{\omega \varepsilon}{2}\right)^{k} \Delta \mathcal{U}_{i}^{k}\left(\frac{\mathbf{x}}{\varepsilon}\right) \\
& +\omega^{2} \sum_{i=0}^{n} \sum_{k=0}^{i}\left(\frac{\omega \varepsilon}{2}\right)^{i}\left(\log \frac{\omega \varepsilon}{2}\right)^{k} \mathcal{U}_{i}^{k}\left(\frac{\mathbf{x}}{\varepsilon}\right)
\end{aligned}\right.
$$


That is to say using the embedded Laplace equations (2.26)

$$
\left\{\begin{aligned}
\left(\Delta u_{N, n}^{\varepsilon}+\omega^{2} u_{N, n}^{\varepsilon}\right)(\mathbf{x})= & -\frac{1}{\varepsilon^{2}} \sum_{i=0}^{n} \sum_{k=0}^{i}\left(\frac{\omega \varepsilon}{2}\right)^{i}\left(\log \frac{\omega \varepsilon}{2}\right)^{k} \mathcal{U}_{i-2}^{k}\left(\frac{\mathbf{x}}{\varepsilon}\right) \\
& +\omega^{2} \sum_{i=0}^{n} \sum_{k=0}^{i}\left(\frac{\omega \varepsilon}{2}\right)^{i}\left(\log \frac{\omega \varepsilon}{2}\right)^{k} \mathcal{U}_{i}^{k}\left(\frac{\mathbf{x}}{\varepsilon}\right)
\end{aligned}\right.
$$

Most of the terms in this sums cancel and it remains

$$
\left(\Delta u_{N, n}^{\varepsilon}+\omega^{2} u_{N, n}^{\varepsilon}\right)(\mathbf{x})=\omega^{2} \sum_{i=n-1}^{n} \sum_{k=0}^{i}\left(\frac{\omega \varepsilon}{2}\right)^{i}\left(\log \frac{\omega \varepsilon}{2}\right)^{k} \mathcal{U}_{i}^{k}\left(\frac{\mathbf{x}}{\varepsilon}\right)
$$

Combining (3.140), (3.141), and (3.144), we obtain with $\mathcal{B}_{H}^{\varepsilon}=\frac{B_{H}^{\varepsilon}}{\varepsilon}$

$$
\left\{\begin{aligned}
\left\|\Delta \widetilde{u}_{n}^{\varepsilon}+\omega^{2} \widetilde{u}_{n}^{\varepsilon}\right\|_{L^{\infty}\left(B_{H}^{\varepsilon}\right)} \leqslant & \frac{C}{\eta_{H}}\left\|\nabla \mathcal{E}_{n}^{\varepsilon}\right\|_{L^{\infty}\left(C_{H}^{\varepsilon}\right)}+\frac{C}{\eta_{H}^{2}}\left\|\mathcal{E}_{n}^{\varepsilon}\right\|_{L^{\infty}\left(C_{H}^{\varepsilon}\right)} \\
& +C\left(\log \frac{\omega \varepsilon}{2}\right)^{n} \sum_{i=n-1}^{n} \sum_{k=0}^{i}\left[\left(\frac{\omega \varepsilon}{2}\right)^{i}\left\|\mathcal{U}_{i}^{k}\right\|_{L^{\infty}\left(\mathcal{B}_{H}^{\varepsilon}\right)}\right]
\end{aligned}\right.
$$

As a consequence of property $(2.29)(\mathrm{i})$ of $\mathcal{U}_{i}^{k}$

$$
\left\|\mathcal{U}_{i}^{k}\right\|_{L^{\infty}\left(\mathcal{B}_{H}^{\varepsilon}\right)} \leqslant C_{i}^{k}\left(\frac{\eta_{H}}{\varepsilon}\right)^{i-k}
$$

we have

$$
\sum_{i=n-1}^{n} \sum_{k=0}^{i}\left[\left(\frac{\omega \varepsilon}{2}\right)^{i}\left\|\mathcal{U}_{i}^{k}\right\|_{L^{\infty}\left(\mathcal{B}_{H}^{\varepsilon}\right)}\right] \leqslant \sum_{i=n-1}^{n} \sum_{k=0}^{i} C_{i, k}\left[\left(\frac{\omega \varepsilon}{2}\right)^{k}\left(\frac{\omega \eta_{H}}{2}\right)^{i-k}\right] \leqslant C_{n} \eta_{H}^{n-1}
$$

where for the last inequality we have used the brute estimate $\varepsilon \leqslant C \eta_{H}(\varepsilon)$.

We deduce the final estimate (3.136)(i) from (3.145), (3.147), and the estimates (3.82) of Lemma 3.6.

The proof of the second estimate (3.136)(ii) follows exactly the same lines and uses the property (2.29)(i) of the function $u_{i}^{k}$. The details are left to the reader.

Proof of Lemma 3.10. We shall restrict ourselves to prove the first estimate (3.137). The proof of the second one (3.138) follows the same lines and is left to the reader.

Let us introduce a cut-off function

$$
\Psi(\mathbf{x})=\psi(|\mathbf{x}|),
$$

where $\psi \in C^{\infty}(R)$ is equal to 1 in the interval $[0, R / 2]$ and vanishes in $[3 R / 4,+\infty[$. We assume that $\varepsilon$ is small enough in order that $\eta_{H}(\varepsilon)<R / 2$. Using polar coordinates, we have

$$
\|v\|_{L^{1}\left(B_{H}^{\varepsilon}\right)}=\|\Psi v\|_{L_{1}\left(B_{H}^{\varepsilon}\right)}=\int_{0}^{2 \eta_{H}(\varepsilon)} \int_{0}^{\pi}|\Psi v(r, \theta)| r \mathrm{~d} r \mathrm{~d} \theta .
$$

Since $\Psi v(R, \theta)=0$, we can write

$$
|\Psi v(r, \theta)|=\left|\int_{r}^{R} \frac{\partial \Psi v}{\partial r}\left(r^{\prime}, \theta\right) \mathrm{d} r^{\prime}\right|
$$


By Cauchy-Schwarz inequality, one has

$$
\left\{\begin{aligned}
|\Psi v(r, \theta)| & \leq\left(\int_{r}^{R}\left|\frac{\partial \Psi v}{\partial r}\left(r^{\prime}, \theta\right)\right|^{2} r^{\prime} \mathrm{d} r^{\prime}\right)^{\frac{1}{2}}\left(\int_{r}^{r_{0}} \frac{1}{r^{\prime}} \mathrm{d} r^{\prime}\right)^{\frac{1}{2}} \\
& =\left[\log \left(\frac{R}{r}\right)\right]^{\frac{1}{2}}\left(\int_{0}^{R}\left|\frac{\partial \Psi v}{\partial r}\left(r^{\prime}, \theta\right)\right|^{2} r^{\prime} \mathrm{d} r^{\prime}\right)^{\frac{1}{2}}
\end{aligned}\right.
$$

Substituting this inequality in (3.149), we get (with $\eta=\eta_{H}(\varepsilon)$ )

$$
\|v\|_{L^{1}\left(B_{H}^{\varepsilon}\right)} \leq \int_{0}^{2 \eta} \int_{0}^{\pi}\left(\int_{0}^{R}\left|\frac{\partial \Psi v}{\partial r}\left(r^{\prime}, \theta\right)\right|^{2} r^{\prime} \mathrm{d} r^{\prime}\right)^{1 / 2}\left[\log \left(\frac{R}{r}\right)\right]^{\frac{1}{2}} r \mathrm{~d} r \mathrm{~d} \theta .
$$

By Cauchy-Schwarz inequality, we obtain (still with $\eta=\eta_{H}(\varepsilon)$ )

$$
\|v\|_{L^{1}\left(B_{H}^{\varepsilon}\right)} \leq\left[\int_{0}^{2 \eta}\left(\int_{0}^{\pi} \int_{0}^{R}\left|\frac{\partial v}{\partial r}\left(r^{\prime}, \theta\right)\right|^{2} r^{\prime} \mathrm{d} r^{\prime} \mathrm{d} \theta\right) r \mathrm{~d} r\right]^{\frac{1}{2}}\left[\int_{0}^{2 \eta} \int_{0}^{\pi} \log \left(\frac{R}{r}\right) r \mathrm{~d} r \mathrm{~d} \theta\right]^{\frac{1}{2}}
$$

which yields immediately

$$
\|v\|_{L^{1}\left(B_{H}^{\varepsilon}\right)} \leq \sqrt{2 \pi} \eta_{H}(\varepsilon)\left(\int_{0}^{2 \eta_{H}(\varepsilon)} \log \left(\frac{R}{r}\right) r \mathrm{~d} r\right)^{\frac{1}{2}}\left\|\frac{\partial(\Psi v)}{\partial r}\right\|_{L^{2}\left(B_{R}\right)}
$$

The final result follows from the inequality $\left\|\frac{\partial(\Psi v)}{\partial r}\right\|_{L^{2}\left(B_{R}\right)} \leq C_{R}\|v\|_{H^{1}\left(B_{R}\right)}$ and the identity

$$
\int_{0}^{2 \eta_{H}(\varepsilon)} \log \left(\frac{R}{r}\right) r \mathrm{~d} r=2 \eta_{H}(\varepsilon)\left(1+\log \left[\frac{R}{2 \eta_{H}(\varepsilon)}\right]\right) .
$$

\section{Conclusion}

In the context of acoustic waves in frequency domain, we have seen that the technique of matching of asymptotic expansions can be applied to derive approximate models for thin slot problems. These models are validated by error estimates obtained by techniques similar to those used for the justification of multiscale expansions.

There remains a lot of open questions. We propose below a (non exhaustive) list of such questions.

- Extension of the 2D analysis. We think that the forthcoming problems should be addressed.

- The case of a slot of finite length. Except for a countable set of lengths of slot (multiples of the half of the wave length), the results are essentially identical to the ones of this article. This work has already been partly achieved and can be found in [4]. The proof is similar except for the "resonances" of the finite slot, see [1], where this problem remains open.

- The case of a curved slot. Provided that the slot remains straight for $0<x<\delta$, this case should be possible to treat thanks to a perturbation analysis with respect to the straight slot case.

- The theory in the case of the Dirichlet condition. In this case, the situation is quite different since the wave which is transmitted in the slot is purely evanescent and the pure half-space solution already provides a much better approximation than for the Neumann condition. 


\section{- Generalizations of the 2D model.}

- The 3D case. There are several geometrical configurations that should be analyzed. We add a third dimension associated with the $z$-direction. The case where the slot is infinite in the $z$-direction is practically equivalent to the $2 \mathrm{D}$ case. However, one can expect different results depending on the fact that the $z$-dimension of the slot is $O(1)$ or $O(\varepsilon)$.

- Maxwell's system. The interesting case would correspond to the perfectly conducting boundary condition. In such a case, one must expect different behaviours of the different components of the electric field since one has to consider either the Dirichlet or Neumann condition depending on the polarization of the electromagnetic field (this is already mentioned in [21]).

\section{REFERENCES}

[1] J. Beale, Scattering frequencies of reasonators. Comm. Pure Appl. Math. 26 (1973) 549-563.

[2] C. Butler and D. Wilton, General analysis of narrow strips and slots. IEEE Trans. Antennas Propag. 28 (1980) 42-48.

[3] G. Caloz, M. Costabel, M. Dauge and G. Vial, Asymptotic expansion of the solution of an interface problem in a polygonal domain with thin layer. Asymptotic Anal. 50 (2006) 121-173.

[4] M. Clausel, M. Duruflé, P. Joly and S. Tordeux, A mathematical analysis of the resonance of the finite thin slots. Appl. Numer. Math. 56 (2006) 1432-1449.

[5] D. Crighton, A. Dowling, J.F. Williams, M. Heckl and F. Leppington, An asymptotic analysis, in Modern Methods in Analytical acoustics, Lecture Notes, Springer-Verlag, London (1992).

[6] J. Gilbert and R. Holland, Implementation of the thin-slot formalism in the finite-difference EMP code THREEDII. IEEE Trans. Nucl. Sci. 28 (1981) 4269-4274.

[7] P. Harrington and D. Auckland, Electromagnetic transmission through narrow slots in thick conducting screens. IEEE Trans. Antennas Propag. 28 (1980) 616-622.

[8] A.M. Il'in, Matching of Asymptotic Expansions of Solutions of Boundary Value Problems, Translations of Mathematical Monographs 102. American Mathematical Society, Providence, RI (1992). Translated from the Russian by V. Minachin [V.V. Minakhin].

[9] P. Joly and S. Tordeux, Asymptotic analysis of an approximate model for time harmonic waves in media with thin slots. ESAIM: M2AN 40 (2006) 63-97.

[10] P. Joly and S. Tordeux, Matching of asymptotic expansions for wave propagation in media with thin slots I: The asymptotic expansion. Multiscale Model. Simul. 5 (2006) 304-336.

[11] G. Kriegsmann, The flanged waveguide antenna: discrete reciprocity and conservation. Wave Motion 29 (1999) 81-95.

[12] N. Lebedev, Special functions and their applications. Revised English edition, translated and edited by Richard A. Silverman, Prentice-Hall Inc., Englewood Cliffs, N.J. (1965).

[13] M. Li, J. Nuebel, J. Drewniak, R. DuBroff, T. Hubing and T. Van Doren, EMI from cavity modes of shielding enclosures-fdtd modeling and measurements. IEEE Trans. Electromagn. Compat. 42 (2000) 29-38.

[14] V. Maz'ya, S. Nazarov and B. Plamenevskii, Asymptotische Theorie elliptischer Randwertaufgaben in singulär gestörten Gebieten I, in Mathematische Monographien, Band 82, Akademie Verlag, Berlin (1991).

[15] V. Maz'ya, S. Nazarov and B. Plamenevskii, Asymptotische Theorie elliptischer Randwertaufgaben in singulär gestörten Gebieten II, in Mathematische Monographien, Band 83, Akademie Verlag, Berlin (1991).

[16] B. Noble, Methods based on the Wiener-Hopf technique for the solution of partial differential equations, International Series of Monographs on Pure and Applied Mathematics 7. Pergamon Press, New York (1958).

[17] O. Oleinik, A. Shamaev and G. Yosifian, Mathematical Problems in Elasticity and Homogenization, Studies in Mathematics and Its Applications. North-Holland, Amsterdam (1992).

[18] S. Schot, Eighty years of Sommerfeld's radiation condition. Historia Math. 19 (1992) 385-401.

[19] A. Taflove, Computational Electrodynamics: The Finite-Difference Time-Domain Method. Artech House Inc., Boston, MA (1995).

[20] A. Taflove, K. Umashankar, B. Becker, F. Harfoush and K. Yee, Detailed fdtd analysis of electromagnetic fields penetrating narrow slots ans lapped joints in thick conducting screens. IEEE Trans. Antennas Propag. 36 (1988) 247-257.

[21] F. Tatout, Propagation d'une onde électromagnétique dans une fente mince. Propagation et réflexion d'ondes en élasticité. Application au contrôle. Ph.D. thesis, École normale supérieure de Cachan, France (1996).

[22] S. Tordeux, Méthodes asymptotiques pour la propagation des ondes dans les milieux comportant des fentes. Ph.D. thesis, Université de Versailles, France (2004).

[23] S. Tordeux and G. Vial, Matching of asymptotic expansions and multiscale expansion for the rounded corner problem. Tech. Rep. 2006-04, ETHZ, Seminar for applied mathematics (2006).

[24] M. Van Dyke, Perturbation methods in fluid mechanics. The Parabolic Press, Stanford, California (1975).

[25] G. Vial, Analyse multiéchelle et conditions aux limites approchées pour un problème de couche mince dans un domaine à coin. Ph.D. thesis, Université de Rennes I, France (2003). 